CRYSTALLOGRAPHIC COMMUNICATIONS

ISSN 2056-9890

Received 15 November 2021

Accepted 9 December 2021

Edited by M. Weil, Vienna University of Technology, Austria

Keywords: crystal structure; thioamide; intraand intermolecular interactions; Hirshfeld surface.

CCDC reference: 2127513

Supporting information: this article has supporting information at journals.iucr.org/e

\section{Crystal structure and Hirshfeld surface analysis of 3-methyl-4-oxo- $N$-phenyl-3,4-dihydroquinazoline- 2-carbothioamide}

\author{
Nasiba Pirnazarova, ${ }^{a}$ Ubaydullo Yakubov, ${ }^{\text {b }}$ Sevara Allabergenova, ${ }^{\text {b }}$ Akmaljon \\ Tojiboev $^{c, d}{ }^{\text {d }}$ Kambarali Turgunov ${ }^{b, e}$ and Burkhon Elmuradov ${ }^{b}$
}

${ }^{a}$ Qarshi State University, Kochabog str. 17, Qarshi 180119, Uzbekistan, ${ }^{\mathbf{b}_{S}}$. Yunusov Institute of Chemistry of Plant Substances, Academy of Sciences of Uzbekistan, Mirzo Ulugbek str. 77, Tashkent 100170, Uzbekistan, ' University of Geological Sciences, Olimlar str. 64, Mirzo Ulugbek district, Tashkent, Uzbekistan, ${ }^{\mathbf{d}}$ National University of Uzbekistan named after Mirzo Ulugbek 100174, University Str. 4, Olmazor District, Tashkent, Uzbekistan, and ${ }^{\mathbf{e}}$ Turin Polytechnic University in Tashkent, Kichik Khalka yuli str. 17, 100095 Tashkent, Uzbekistan. *Correspondence e-mail: a_tojiboev@yahoo.com

The asymmetric unit of the title compound, $\mathrm{C}_{16} \mathrm{H}_{13} \mathrm{~N}_{3} \mathrm{OS}$, comprises two molecules $(A$ and $B)$ with similar conformations that differ mainly in the orientation of the phenyl group relative to the rest of the molecule, as expressed by the $\mathrm{C}_{\text {thioamide }}-\mathrm{N}_{\text {thioamide }}-\mathrm{C}_{\text {phenyl }}-\mathrm{C}_{\text {phenyl }}$ torsion angle of $49.3(3)^{\circ}$ for molecule $A$ and of $5.4(3)^{\circ}$ for molecule $B$. In the crystal, two intermolecular N$\mathrm{H} \cdots \mathrm{N}$ hydrogen bonds lead to the formation of a dimer with $R_{2}^{2}(10)$ graph-set notation. A Hirshfeld surface analysis revealed that $\mathrm{H} \cdots \mathrm{H}$ interactions are the most important intermolecular interactions, contributing $40.9 \%$ to the Hirshfeld surface.

\section{Chemical context}

Thioamides and their derivatives are important representatives of organic compounds containing a sulfur atom. The presence of bifunctional properties in thioamides, resulting from the presence of nitrogen and sulfur atoms, and their participation in reactions as electrophilic or nucleophilic reagents can lead to the formation of different heterocyclic compounds. Several review articles have been published on the syntheses, physico-chemical properties and applications of thioamides (Jagodziński, 2003; Belskaya et al., 2010; Koketsu \& Ishihara, 2007; Krayushkin et al., 2004; Britsun et al., 2008).

One of the methods of choice for the synthesis of widely used thioamides is the Wilgerodt-Kindler reaction. As shown by previous studies, the Wilgerodt-Kindler reactions with 2-methylquinazoline-4-one went to the active methyl group in the position 2 and, accordingly, thioamides were synthesized in a series of quinazoline derivatives (Shakhidoyatov et al., 1997). Continuing our work in this direction, we have synthesized 2,3-dimethylquinazoline-4-one and studied the corresponding Wilgerodt-Kindler reactions.

During the reaction involving 2,3-dimethylquinazoline-4one, sulfur, aniline, the solvent dimethyl sulfoxide and the catalyst sodium sulfide, the reaction went to the active methyl group in position 2 and new thioamides of a number of derivatives of quinazoline-4-one were obtained. The synthesis and crystal structure of 3-methyl-4-oxo- $\mathrm{N}$-phenyl-3,4-dihydroquinazoline-2-carbothioamide, $\mathrm{C}_{16} \mathrm{H}_{13} \mathrm{~N}_{3} \mathrm{OS}$, is reported here. Relevant intermolecular contacts were quantified by using Hirshfeld surface analysis. 
<smiles>Cn1c(C(=S)Nc2ccccc2)nc2ccccc2c1=O</smiles>

\section{Structural commentary}

The title compound crystallizes with two molecules, $A$ and $B$, in the asymmetric unit (Fig. 1). In molecules $A$ and $B$ the orientations of the quinazoline ring system and the phenyl ring relative to the thioamide group differ, as shown by the values of the $\mathrm{N} 3-\mathrm{C} 2-\mathrm{C} 10-\mathrm{S} 1$ and $\mathrm{C} 10-\mathrm{N} 11-\mathrm{C} 12-\mathrm{C} 13$ torsion angles of 76.14 (19) and $49.3(3)^{\circ}$, respectively, in molecule $A$

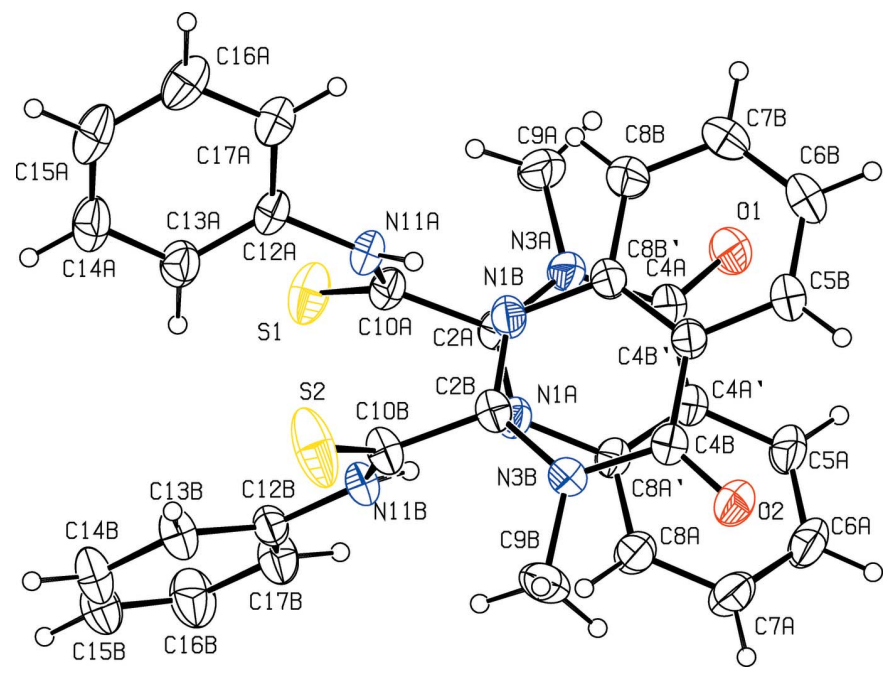

Figure 1

Asymmetric unit of the title compound with the atom-numbering scheme. Displacement ellipsoids for non-hydrogen atoms are drawn at the $30 \%$ probability level.

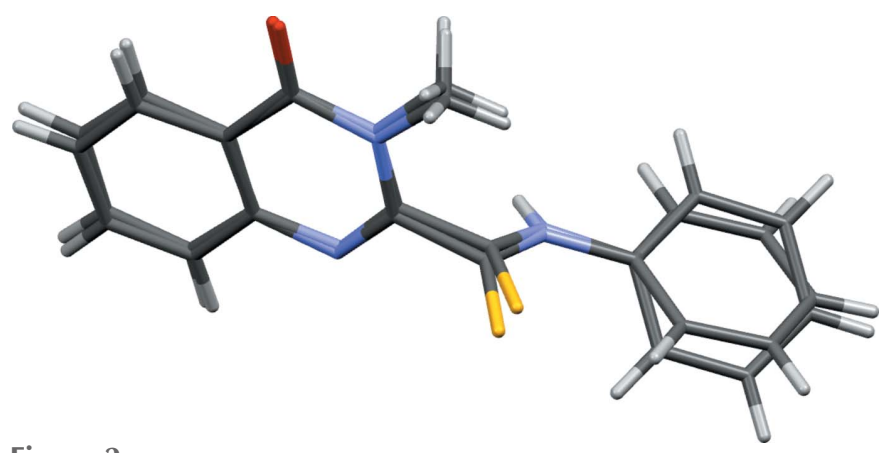

Figure 2

Overlay plot of the two independent molecules in the title compound.
Table 1

Hydrogen-bond geometry $\left(\AA,^{\circ}\right)$.

\begin{tabular}{lllll}
\hline$D-\mathrm{H} \cdots A$ & $D-\mathrm{H}$ & $\mathrm{H} \cdots A$ & $D \cdots A$ & $D-\mathrm{H} \cdots A$ \\
\hline $\mathrm{N} 1 A-\mathrm{H} 11 A \cdots \mathrm{N} 1 B$ & $0.88(2)$ & $2.05(2)$ & $2.913(2)$ & $166.7(18)$ \\
$\mathrm{N} 1 B-\mathrm{H} 11 B \cdots \mathrm{N} 1 A$ & $0.87(2)$ & $2.04(2)$ & $2.907(2)$ & $171.6(19)$ \\
$\mathrm{C} 9 A-\mathrm{H} 9 A B \cdots \mathrm{S} 1 A$ & 0.96 & 2.87 & $3.424(2)$ & 118 \\
$\mathrm{C} 13 B-\mathrm{H} 13 B \cdots \mathrm{S} 1 B$ & 0.93 & 2.58 & $3.243(3)$ & 129 \\
$\mathrm{C} 7 A-\mathrm{H} 7 A \cdots \mathrm{O} 1 B^{\mathrm{i}}$ & 0.93 & 2.49 & $3.386(3)$ & 162 \\
$\mathrm{C} 7 B-\mathrm{H} 7 B \cdots \mathrm{O} 1 A^{\mathrm{ii}}$ & 0.93 & 2.47 & $3.385(3)$ & 166 \\
\hline
\end{tabular}

Symmetry codes: (i) $-x+1,-y+2,-z+1$; (ii) $-x+1,-y+1,-z+1$.

and $83.78(19)$ and $5.4(3)^{\circ}$ in molecule $B$. As a result, there are differences in the intramolecular distances between the sulfur and hydrogen atoms in molecules $A$ and $B$. In molecule $A$, the contacts $\mathrm{S} 1 A \cdots \mathrm{H} 9 A B$ and $\mathrm{S} 1 A \cdots \mathrm{H} 13 A$ are 2.873 and $2.897 \AA$ whereas the corresponding distances in molecule $B$ are 3.054 and $2.578 \AA$. The phenyl and pyrimidine rings in both molecules are essentially coplanar, with r.m.s. deviations of 0.0225 and $0.0119 \AA$ for molecule $A$ and $B$, respectively. Fig. 2 shows that the pyrimidine moieties of the molecules are almost superimposable.

\section{Supramolecular features}

In the crystal, molecules $A$ and $B$ form a dimer with an $R_{2}^{2}(10)$ ring motif through intermolecular $\mathrm{N}-\mathrm{H} \cdots \mathrm{N}$ hydrogen bonds (Fig. 3, Table 1). In addition, molecule $A$ interacts with molecule $B$ by a $\mathrm{C}-\mathrm{H} \cdots \pi$ interaction (the $\mathrm{C} 13 A-\mathrm{H} \cdots C g 1$ distance is $3.148 \AA, C g 1$ is the centroid of atoms $\mathrm{C} 12 B-\mathrm{C} 17 B)$. Other weak $\mathrm{C} 7 A-\mathrm{H} 7 A \cdots \mathrm{O} 1 B, \mathrm{C} 7 A-\mathrm{H} 7 A \cdots \mathrm{O} 1 B, \mathrm{C} 7 B-$ $\mathrm{H} 7 B \cdots \mathrm{O} 1 A, \mathrm{C} 9 A-\mathrm{H} 9 A B \cdots \mathrm{S} 1 A$ and $\mathrm{C} 13 B-\mathrm{H} 13 B \cdots \mathrm{S} 1 B$ hydrogen bonds link adjacent dimers, forming supramolecular layers expanding parallel to (010) (Fig. 4). The overall packing of molecules leads to the formation of narrow channels along

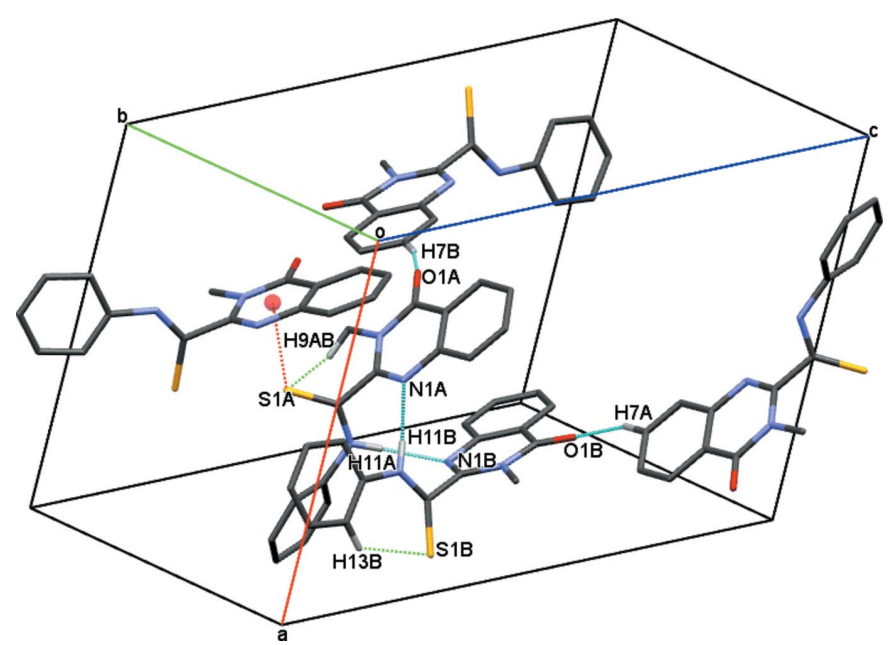

Figure 3

A diagram showing the intramolecular $\mathrm{C}-\mathrm{H} \cdots \mathrm{S}$ (green dashed lines) and the intermolecular $\mathrm{N}-\mathrm{H} \cdots \mathrm{N}$ (light blue dashed lines) and $\mathrm{C}-$ $\mathrm{H} \cdots \mathrm{O}$ (blue dashed lines) hydrogen bonds, as well as $\mathrm{C}-\mathrm{S} \cdots \pi$ (red dashed lines) interactions present in the title compound. $\mathrm{H}$ atoms not involved in the interactions have been omitted for clarity. 


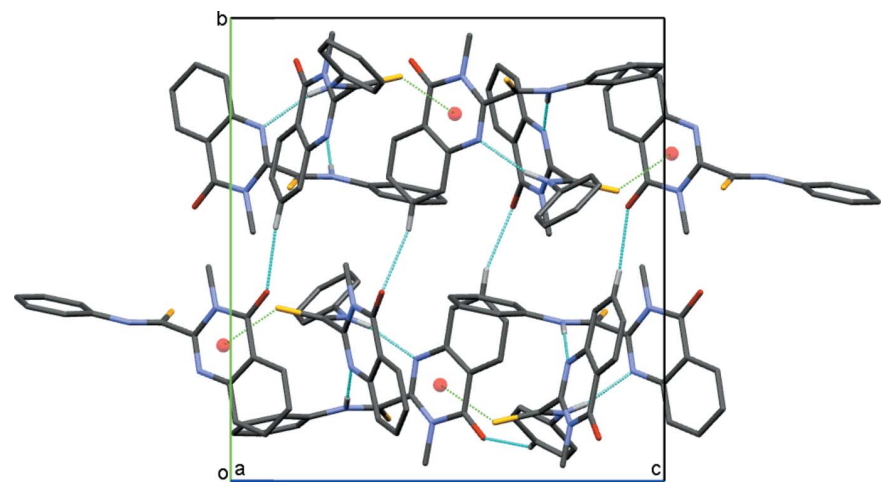

Figure 4

A view of the crystal packing of the title compound along the $a$ axis. Intermolecular hydrogen bonds and $\mathrm{C}-\mathrm{S} \cdots \pi$ interactions are displayed by blue and green dotted lines, respectively.

the $b$-axis direction, passing through nodes and the centre of the cell (Fig. 5).

\section{Hirshfeld surface analysis}

A Hirshfeld surface (HS) analysis (Spackman \& Jayatilaka, 2009) was carried out using CrystalExplorer17.5 (Turner et al., 2017) to quantify and visualize intermolecular interactions in the crystal structure of the title compound. The HS mapped with $d_{\text {norm }}$ is represented in Fig. 6 . The white surface indicates contacts with distances equal to the sum of van der Waals radii, and the red and blue colours indicate distances shorter or longer, respectively, than the van der Waals radii. The twodimensional fingerprint plot for all contacts is depicted in Fig. $7 a$, and delineated in $\mathrm{H} \cdots \mathrm{H}, \mathrm{C} \cdots \mathrm{H} / \mathrm{H} \cdots \mathrm{C}, \mathrm{S} \cdots \mathrm{H} / \mathrm{H} \cdots \mathrm{S}$, $\mathrm{N} \cdots \mathrm{H} / \mathrm{H} \cdots \mathrm{N}$, and $\mathrm{O} \cdots \mathrm{H} / \mathrm{H} \cdots \mathrm{O}$ contacts (Fig. $7 b-f$ ) whereby $\mathrm{H} \cdot \mathrm{H}$ contacts are responsible for the largest contribution $(40.9 \%)$ to the Hirshfeld surface. C $\cdots \mathrm{H} / \mathrm{H} \cdots \mathrm{C}$ contribute $23.7 \%, \mathrm{~S} \cdots \mathrm{H} / \mathrm{H} \cdots \mathrm{S}$ contacts $10.7 \%, \mathrm{~N} \cdots \mathrm{H} / \mathrm{H} \cdots \mathrm{N}$ contacts $8.1 \%$ and $\mathrm{O} \cdots \mathrm{H} / \mathrm{H} \cdots \mathrm{O}$ contacts $7.0 \%$ to the total Hirshfeld surface. The contributions of further contacts are only minor and amount to $\mathrm{C} \cdots \mathrm{C}(4.0 \%), \mathrm{S} \cdots \mathrm{C} / \mathrm{C} \cdots \mathrm{S}(1.9 \%), \mathrm{N} \cdots \mathrm{C} /$

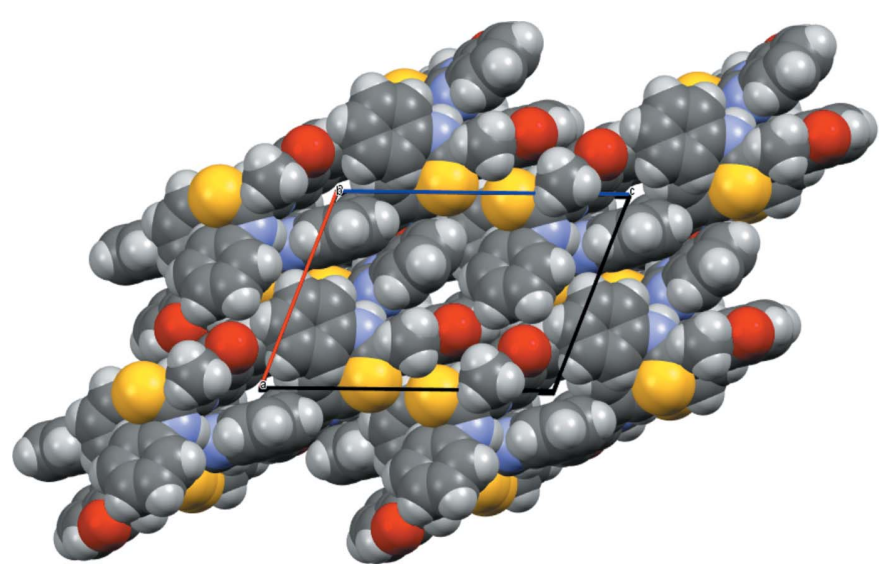

Figure 5

View of the narrow channels formed along the $b$ axis.

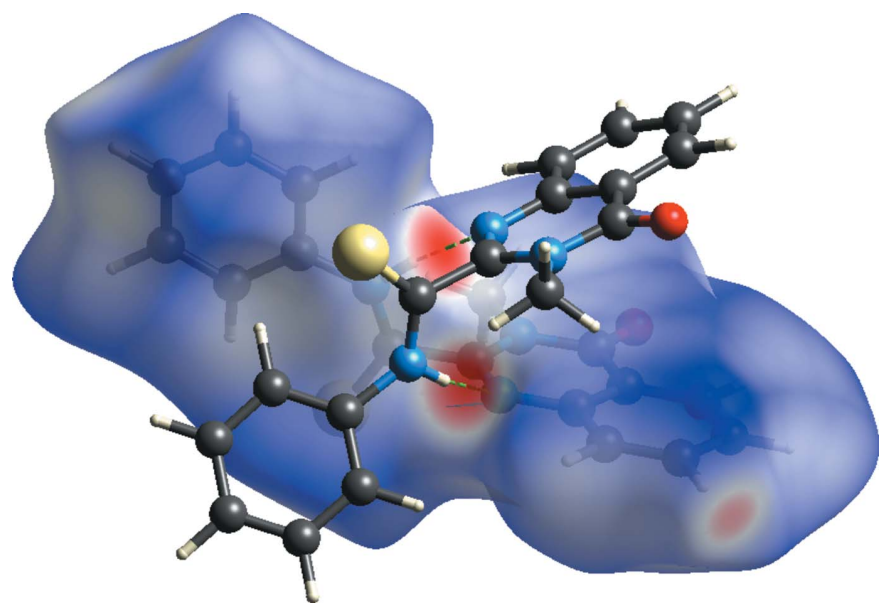

Figure 6

View of the three-dimensional Hirshfeld surface of the title compound plotted over $d_{\text {norm }}$.

$\mathrm{C} \cdots \mathrm{N}(1.2 \%), \mathrm{S} \cdots \mathrm{S}(1.0 \%), \mathrm{S} \cdots \mathrm{C} / \mathrm{C} \cdots \mathrm{S}(0.6 \%), \mathrm{O} \cdots \mathrm{N} /$ $\mathrm{N} \cdots \mathrm{O}(0.2 \%)$ and $\mathrm{O} \cdots \mathrm{C} / \mathrm{C} \cdots \mathrm{O}(0.1 \%)$.

\section{Database survey}

A search in the Cambridge Structural Database (CSD, version 5.41, update of January 2020; Groom et al., 2016) revealed six matches for molecules containing the 2,3-dimethylquinazolin$4(3 H)$-one moiety with a similar planar conformation as that in the title structure: AFOCIJ (Utayeva et al., 2013), HOCYED (Voitenko et al., 1999), MAHLOZ (Kotipalli et al., 2016), MUDHIE (Baglai et al., 2014), UTIDIM (Kundu et al., 2016) and XODZIB (Saitkulov et al., 2014). A search for the 2-methyl- $N$-phenylprop-2-enethioamide moiety gave six hits: ADEKUQ (Xiao \& Jian, 2006), AGECIB (Skelton \& Massi, 2018), GOFFOY (Li et al., 2014), GOXFUW (Li et al., 2016),
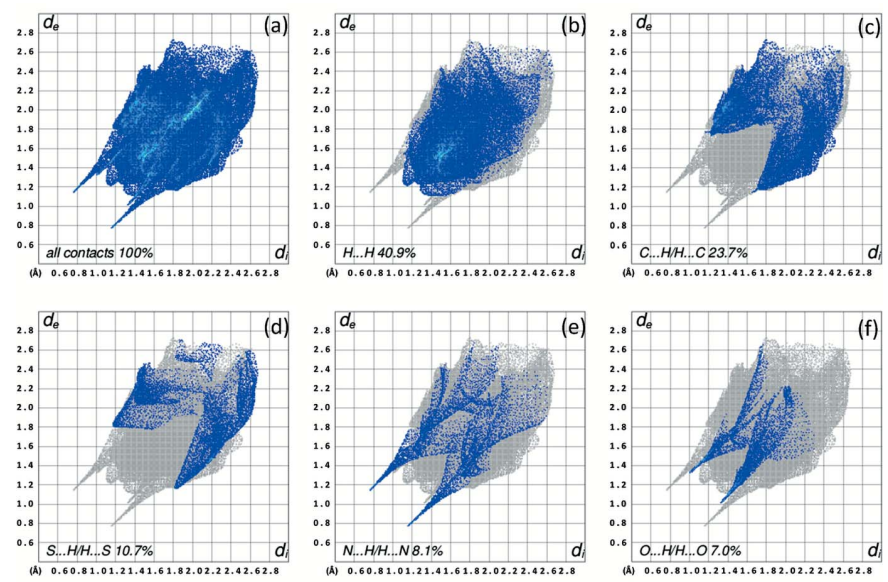

Figure 7

Two-dimensional fingerprint plots for the title compound, $(a)$ for all contacts and delineated into $(b) \mathrm{H} \cdots \mathrm{H},(c) \mathrm{C} \cdots \mathrm{H} / \mathrm{H} \cdots \mathrm{C},(d) \mathrm{S} \cdots \mathrm{H} /$ $\mathrm{H} \cdots \mathrm{S},(e) \mathrm{N} \cdots \mathrm{H} / \mathrm{H} \cdots \mathrm{N}$ and $(f) \mathrm{O} \cdots \mathrm{H} / \mathrm{H} \cdots \mathrm{O}$ contacts. $d_{\mathrm{i}}$ and $d_{\mathrm{e}}$ denote the closest internal and external distances (in $\AA$ ) from a point on the surface. 
JURWEA (Guo et al., 2015) and QAJVAY (Mereiter et al., 2000).

\section{Synthesis and crystallization}

$0.435 \mathrm{~g}(0.0025 \mathrm{~mol})$ of 2,3-dimethylquinazoline-4-one, $0.465 \mathrm{~g}$ $(0.005 \mathrm{~mol})$ of aniline, $0.24 \mathrm{~g}(0.0075 \mathrm{~mol})$ of sulfur, $0.05 \mathrm{~g}$ of sodium sulfide $\left(\mathrm{Na}_{2} \mathrm{~S} \cdot 9 \mathrm{H}_{2} \mathrm{O}\right)$ and $4 \mathrm{ml}$ of dimethyl sulfoxide were injected into a round-bottomed flask with a volume of $100 \mathrm{ml}$. Then the reaction flask was heated to $403 \mathrm{~K}$ for $6 \mathrm{~h}$. After the end of the reaction, the flask was cooled and $40 \mathrm{ml}$ of an aqueous sodium hydroxide solution were added. The resulting mixture was filtered, then added to a dilute solution of sulfuric acid ( $\mathrm{pH} 6$ ). The formed precipitate was filtered off and recrystallized in methanol. In total, $0.5 \mathrm{~g}(64.0 \%)$ of the product were obtained, m.p. 481-483 K.

\section{Refinement}

Crystal data, data collection and structure refinement details are summarized in Table 2. C-bound $\mathrm{H}$ atoms were positioned geometrically, with $\mathrm{C}-\mathrm{H}=0.96 \AA$ (for methylene $\mathrm{H}$ atoms) and $\mathrm{C}-\mathrm{H}=0.93 \AA$ (for aromatic $\mathrm{H}$ atoms), and were refined with $U_{\text {iso }}(\mathrm{H})=1.5 U_{\text {eq }}\left(\mathrm{C}_{\text {methyl }}\right)$ and $1.2 U_{\text {eq }}(\mathrm{C})$, respectively. $\mathrm{H}$ atoms bonded to nitrogen were located in a difference-Fourier map, and their positional and isotropic displacement parameters were freely refined.

\section{Acknowledgements}

The authors are grateful to the Institute of Bioorganic Chemistry, Academy Sciences of Uzbekistan, for providing laboratory facilities.

\section{Funding information}

The chemical part of the work was financially supported by a Georg Forster Research Fellowship for Experienced Researchers of the Alexander von Humboldt Foundation $(\mathrm{AvH})$ to BE (UZB $1186936 \mathrm{GF}-\mathrm{E})$.

\section{References}

Baglai, I., Maraval, V., Voitenko, Z. V., Duhayon, C., Volovenko, Y. M. \& Chauvin, R. (2014). Turk. J. Chem. 38, 121-126.

Belskaya, N. P., Dehaen, W. \& Bakulev, V. A. (2010). Arkivoc, pp. 275-332.

Britsun, V. N., Esipenko, A. N. \& Lozinskii, M. O. (2008). Chem. Heterocycl. Compd, 44, 1429-1459.

Groom, C. R., Bruno, I. J., Lightfoot, M. P. \& Ward, S. C. (2016). Acta Cryst. B72, 171-179.

Guo, W. S., Xin, X., Zhao, K. L., Wen, L. R. \& Li, M. (2015). RSC Adv. 5, 70429-70432.

Jagodziński, T. S. (2003). Chem. Rev. 103, 197-228.

Koketsu, M. \& Ishihara, H. (2007). Synthesis, 4, 15-25.

Kotipalli, T., Kavala, V., Janreddy, D., Bandi, V., Kuo, Ch.-W. \& Yao, Ch.-F. (2016). Eur. J. Org. Chem. pp. 1182-1193.

Krayushkin, M. M., Yarovenko, V. N. \& Zavarzin, I. V. (2004). Russ. Chem. Bull. 53, 517-527.

Kundu, P., Mondal, A. \& Chowdhury, C. (2016). J. Org. Chem. 81, 6596-6608.

Li, H. Zh., Xue, W. J. \& Wu, A. X. (2014). Tetrahedron, 70, 4645-4651.
Table 2

Experimental details.

\begin{tabular}{|c|c|}
\hline \multicolumn{2}{|l|}{ Crystal data } \\
\hline Chemical formula & $\mathrm{C}_{16} \mathrm{H}_{13} \mathrm{~N}_{3} \mathrm{OS}$ \\
\hline$M_{\mathrm{r}}$ & 295.35 \\
\hline Crystal system, space group & Monoclinic, $P 2_{1} / n$ \\
\hline Temperature $(\mathrm{K})$ & 566 \\
\hline$a, b, c(\AA)$ & $\begin{array}{l}11.7685(3), 16.3641(3), \\
16.3798(3)\end{array}$ \\
\hline$\beta\left({ }^{\circ}\right)$ & $110.646(2)$ \\
\hline$V\left(\mathrm{\AA}^{6}\right)^{3}$ & $2951.85(11)$ \\
\hline$Z$ & 8 \\
\hline Radiation type & $\mathrm{Cu} K \alpha$ \\
\hline$\mu\left(\mathrm{mm}^{-1}\right)$ & 1.96 \\
\hline Crystal size $(\mathrm{mm})$ & $0.25 \times 0.23 \times 0.20$ \\
\hline \multicolumn{2}{|l|}{ Data collection } \\
\hline Diffractometer & $\begin{array}{l}\text { XtaLAB Synergy, Single source at } \\
\text { home/near, HyPix } 3000\end{array}$ \\
\hline Absorption correction & $\begin{array}{l}\text { Multi-scan (CrysAlis PRO; Rigaku } \\
\text { OD, 2020) }\end{array}$ \\
\hline$T_{\min }, T_{\max }$ & $0.639,1.000$ \\
\hline $\begin{array}{l}\text { No. of measured, independent and } \\
\text { observed }[I>2 \sigma(I)] \text { reflections }\end{array}$ & $16801,5685,4788$ \\
\hline$R_{\text {int }}$ & 0.022 \\
\hline$(\sin \theta / \lambda)_{\max }\left(\AA^{-1}\right)$ & 0.615 \\
\hline \multicolumn{2}{|l|}{ Refinement } \\
\hline$R\left[F^{2}>2 \sigma\left(F^{2}\right)\right], w R\left(F^{2}\right), S$ & $0.042,0.121,1.06$ \\
\hline No. of reflections & 5685 \\
\hline No. of parameters & 390 \\
\hline $\mathrm{H}$-atom treatment & $\begin{array}{l}\mathrm{H} \text { atoms treated by a mixture of } \\
\text { independent and constrained } \\
\text { refinement }\end{array}$ \\
\hline$\Delta \rho_{\max }, \Delta \rho_{\min }\left(\mathrm{e} \AA^{-3}\right)$ & $0.32,-0.43$ \\
\hline
\end{tabular}

Computer programs: CrysAlis PRO (Rigaku OD, 2020), SHELXT (Sheldrick, 2015a), SHELXL (Sheldrick, 2015b), PLATON (Spek, 2020), Mercury (Macrae et al., 2020) and publCIF (Westrip, 2010).

Li, M., Sun, K. N. \& Wen, L. R. (2016). RSC Adv. 6, 21535-21539.

Macrae, C. F., Sovago, I., Cottrell, S. J., Galek, P. T. A., McCabe, P., Pidcock, E., Platings, M., Shields, G. P., Stevens, J. S., Towler, M. \& Wood, P. A. (2020). J. Appl. Cryst. 53, 226-235.

Mereiter, K., Gaith, A. H. \& Fröhlich, J. (2000). Private communication (refcode: QAJVAY). CCDC, Cambridge, England.

Rigaku OD (2020). CrysAlis PRO. Rigaku Oxford Diffraction, Yarnton, England.

Saitkulov, F. E., Tashniyazov, A. A., Mamadrahimov, A. A. \& Shakhidoyatov, K. M. (2014). Acta Cryst. E70, o788.

Shakhidoyatov, Kh. M., Egamov, D. I. \& Askarov, I. R. (1997). Uzbek Chem. J, 6, 26-27.

Sheldrick, G. M. (2015a). Acta Cryst. A71, 3-8.

Sheldrick, G. M. (2015b). Acta Cryst. C71, 3-8.

Skelton, B. W. \& Massi, M. (2018). Private communication (refcode: AGECIB). CCDC, Cambridge, England.

Spackman, M. A. \& Jayatilaka, D. (2009). CrystEngComm, 11, 19-32. Spek, A. L. (2020). Acta Cryst. E76, 1-11.

Turner, M. J., McKinnon, J. J., Wolff, S. K., Grimwood, D. J., Spackman, P. R., Jayatilaka, D. \& Spackman, M. A. (2017). CrystalExplorer17. University of Western Australia. http://hirshfeldsurface.net.

Utayeva, F. R., Okmanov, R. Y., Mukarramov, N. I., Shakhidoyatov, K. M. \& Tashkhodjaev, B. (2013). Acta Cryst. E69, o1094.

Voitenko, Z. V., Samoilenko, V. P., Kovtunenko, V. A., Gurkevich, V. Yu., Tyltin, A. K., Shcherbakov, M. V. \& Shishkin, O. V. (1999). Chem. Heterocycl. Compd. 35, 600-607.

Westrip, S. P. (2010). J. Appl. Cryst. 43, 920-925.

Xiao, H.-L. \& Jian, F.-F. (2006). Acta Cryst. E62, o2854-o2855. 


\section{supporting information}

Acta Cryst. (2022). E78, 47-50 [https://doi.org/10.1107/S2056989021013116]

Crystal structure and Hirshfeld surface analysis of 3-methyl-4-oxo-Nphenyl-3,4-dihydroquinazoline-2-carbothioamide

Nasiba Pirnazarova, Ubaydullo Yakubov, Sevara Allabergenova, Akmaljon Tojiboev, Kambarali Turgunov and Burkhon Elmuradov

Computing details

Data collection: CrysAlis PRO (Rigaku OD, 2020); cell refinement: CrysAlis PRO (Rigaku OD, 2020); data reduction: CrysAlis PRO (Rigaku OD, 2020); program(s) used to solve structure: SHELXT (Sheldrick, 2015a); program(s) used to refine structure: SHELXL (Sheldrick, 2015b); molecular graphics: PLATON (Spek, 2020), Mercury (Macrae et al., 2020); software used to prepare material for publication: publCIF (Westrip, 2010).

3-Methyl-4-oxo-N-phenyl-3,4-dihydroquinazoline-2-carbothioamide

Crystal data

$\mathrm{C}_{16} \mathrm{H}_{13} \mathrm{~N}_{3} \mathrm{OS}$

$M_{r}=295.35$

Monoclinic, $P 2{ }_{1} / n$

$a=11.7685(3) \AA$

$b=16.3641(3) \AA$

$c=16.3798(3) \AA$

$\beta=110.646(2)^{\circ}$

$V=2951.85(11) \AA^{3}$

$Z=8$

\section{Data collection}

XtaLAB Synergy, Single source at home/near, HyPix 3000 diffractometer

Radiation source: micro-focus sealed X-ray tube Detector resolution: 10.00000 pixels $\mathrm{mm}^{-1}$ $\omega$ scans

Absorption correction: multi-scan (CrysAlisPro; Rigaku OD, 2020)

$T_{\min }=0.639, T_{\max }=1.000$

Refinement

Refinement on $F^{2}$

Least-squares matrix: full

$R\left[F^{2}>2 \sigma\left(F^{2}\right)\right]=0.042$

$w R\left(F^{2}\right)=0.121$

$S=1.06$

5685 reflections

390 parameters
$F(000)=1232$

$D_{\mathrm{x}}=1.329 \mathrm{Mg} \mathrm{m}^{-3}$

$\mathrm{Cu} K \alpha$ radiation, $\lambda=1.54184 \AA$

Cell parameters from 9141 reflections

$\theta=2.7-71.1^{\circ}$

$\mu=1.96 \mathrm{~mm}^{-1}$

$T=566 \mathrm{~K}$

Prismatic, yellow

$0.25 \times 0.23 \times 0.20 \mathrm{~mm}$

16801 measured reflections

5685 independent reflections

4788 reflections with $I>2 \sigma(I)$

$R_{\text {int }}=0.022$

$\theta_{\text {max }}=71.4^{\circ}, \theta_{\min }=4.0^{\circ}$

$h=-14 \rightarrow 14$

$k=-19 \rightarrow 17$

$l=-19 \rightarrow 20$

0 restraints

Primary atom site location: structure-invariant direct methods

Hydrogen site location: mixed

$\mathrm{H}$ atoms treated by a mixture of independent and constrained refinement 
$w=1 /\left[\sigma^{2}\left(F_{\mathrm{o}}^{2}\right)+(0.0602 P)^{2}+0.6163 P\right]$

where $P=\left(F_{\mathrm{o}}^{2}+2 F_{\mathrm{c}}^{2}\right) / 3$

$(\Delta / \sigma)_{\max }=0.001$

$\Delta \rho_{\max }=0.32$ e $\AA^{-3}$
$\Delta \rho_{\min }=-0.42$ e $\AA^{-3}$

Extinction correction: SHELXL (Sheldrick, 2015a), $\mathrm{Fc}^{*}=\mathrm{kFc}\left[1+0.001 \mathrm{xFc}^{2} \lambda^{3} / \sin (2 \theta)\right]^{-1 / 4}$

Extinction coefficient: $0.00124(12)$

Special details

Geometry. All esds (except the esd in the dihedral angle between two 1.s. planes) are estimated using the full covariance matrix. The cell esds are taken into account individually in the estimation of esds in distances, angles and torsion angles; correlations between esds in cell parameters are only used when they are defined by crystal symmetry. An approximate (isotropic) treatment of cell esds is used for estimating esds involving l.s. planes.

Fractional atomic coordinates and isotropic or equivalent isotropic displacement parameters $\left(\AA^{2}\right)$

\begin{tabular}{|c|c|c|c|c|}
\hline & $x$ & $y$ & $z$ & $U_{\text {iso }} * / U_{\text {eq }}$ \\
\hline S1A & $0.46771(5)$ & 0.62649 (4) & $0.89046(3)$ & 0.07206 (19) \\
\hline $\mathrm{O} 1 \mathrm{~A}$ & $0.69766(15)$ & $0.59324(9)$ & 0.65155 (11) & $0.0755(4)$ \\
\hline N1A & $0.48752(12)$ & $0.75527(8)$ & $0.72159(9)$ & 0.0456 \\
\hline N1B & $0.22774(13)$ & $0.73699(9)$ & $0.57212(9)$ & 0.0474 \\
\hline S1B & $0.04219(5)$ & $0.86597(6)$ & $0.63642(4)$ & 0.1030 \\
\hline O1B & $0.30121(15)$ & $0.90703(8)$ & $0.41700(10)$ & $0.0690(4)$ \\
\hline $\mathrm{C} 2 \mathrm{~A}$ & 0.49005 (14) & $0.67801(10)$ & $0.73853(10)$ & 0.0430 \\
\hline $\mathrm{C} 2 \mathrm{~B}$ & $0.22279(15)$ & $0.81489(10)$ & $0.58376(10)$ & $0.0444(4)$ \\
\hline N3A & $0.55988(13)$ & $0.62176(8)$ & $0.71623(10)$ & $0.0487(3)$ \\
\hline N3B & $0.24924(13)$ & $0.87402(8)$ & $0.53417(9)$ & 0.0461 \\
\hline $\mathrm{C} 4 \mathrm{~A}$ & $0.63788(17)$ & $0.64470(11)$ & $0.67217(12)$ & $0.0523(4)$ \\
\hline C4B & $0.28038(16)$ & $0.85368(11)$ & $0.46179(11)$ & $0.0476(4)$ \\
\hline $\mathrm{C} 4 \mathrm{~A}^{\prime}$ & $0.63881(16)$ & $0.73161(11)$ & $0.65427(12)$ & 0.0488 \\
\hline $\mathrm{C}^{\prime} \mathrm{B}^{\prime}$ & $0.28702(15)$ & $0.76640(10)$ & $0.44719(10)$ & $0.0441(4)$ \\
\hline C5A & $0.7150(2)$ & $0.76334(13)$ & $0.61289(15)$ & $0.0663(5)$ \\
\hline H5A & 0.767186 & 0.728822 & 0.597752 & $0.080 *$ \\
\hline C5B & $0.31931(18)$ & $0.73805(12)$ & $0.37776(12)$ & $0.0572(5)$ \\
\hline H5B & 0.336243 & 0.775028 & 0.340470 & $0.069 *$ \\
\hline C6A & $0.7128(2)$ & $0.84521(14)$ & $0.59469(16)$ & $0.0719(6)$ \\
\hline H6A & 0.763410 & 0.866124 & 0.567158 & $0.086^{*}$ \\
\hline C6B & $0.3260(2)$ & $0.65590(14)$ & $0.36481(14)$ & $0.0699(6)$ \\
\hline H6B & 0.347755 & 0.637057 & 0.318813 & $0.084 *$ \\
\hline C7A & $0.6353(2)$ & $0.89685(13)$ & $0.61719(15)$ & $0.0669(5)$ \\
\hline H7A & 0.633301 & 0.952178 & 0.603750 & $0.080 *$ \\
\hline C7B & $0.3002(2)$ & $0.60060(13)$ & $0.42011(16)$ & $0.0738(6)$ \\
\hline H7B & 0.304581 & 0.544836 & 0.410662 & $0.089 *$ \\
\hline C8A & $0.56154(18)$ & $0.86710(11)$ & $0.65916(14)$ & $0.0570(5)$ \\
\hline H8A & 0.510698 & 0.902433 & 0.674732 & $0.068 *$ \\
\hline $\mathrm{C} 8 \mathrm{~B}$ & $0.2684(2)$ & $0.62723(12)$ & $0.48872(14)$ & $0.0644(5)$ \\
\hline H8B & 0.251967 & 0.589664 & 0.525684 & $0.077 *$ \\
\hline $\mathrm{C} 8 \mathrm{~A}^{\prime}$ & $0.56261(15)$ & $0.78398(10)$ & $0.67853(11)$ & $0.0443(4)$ \\
\hline $\mathrm{C}^{\prime} \mathrm{B}^{\prime}$ & $0.26094(16)$ & $0.71106(10)$ & $0.50270(11)$ & $0.0455(4)$ \\
\hline C9A & $0.5550(2)$ & $0.53429(12)$ & $0.73431(16)$ & $0.0712(6)$ \\
\hline H9AA & 0.543485 & 0.503622 & 0.682028 & $0.107 *$ \\
\hline H9AB & 0.488631 & 0.524014 & 0.754066 & $0.107 *$ \\
\hline
\end{tabular}




$\begin{array}{lllll}\text { H9AC } & 0.629728 & 0.517988 & 0.778655 & 0.107^{*} \\ \text { C9B } & 0.2411(2) & 0.96167(12) & 0.55107(14) & 0.0673(5) \\ \text { H9BA } & 0.308740 & 0.989779 & 0.544197 & 0.101^{*} \\ \text { H9BB } & 0.242392 & 0.969168 & 0.609523 & 0.101^{*} \\ \text { H9BC } & 0.166757 & 0.983288 & 0.510518 & 0.101^{*} \\ \text { C10A } & 0.40897(15) & 0.64886(10) & 0.78644(11) & 0.0464(4) \\ \text { C10B } & 0.18531(16) & 0.84207(12) & 0.65864(11) & 0.0520(4) \\ \text { N11A } & 0.29256(13) & 0.64598(9) & 0.73505(9) & 0.0459(3) \\ \text { N11B } & 0.27899(14) & 0.84235(9) & 0.73404(9) & 0.0458(3) \\ \text { H11A } & 0.2709(18) & 0.6657(12) & 0.6816(13) & 0.056(5)^{*} \\ \text { H11B } & 0.3453(19) & 0.8211(12) & 0.7308(13) & 0.060(6)^{*} \\ \text { C12A } & 0.19183(15) & 0.62418(10) & 0.76017(11) & 0.0458(4) \\ \text { C12B } & 0.28641(16) & 0.86210(10) & 0.82016(10) & 0.0460(4) \\ \text { C13A } & 0.17430(18) & 0.65831(12) & 0.83199(13) & 0.0579(5) \\ \text { H13A } & 0.231162 & 0.694273 & 0.868105 & 0.070^{*} \\ \text { C13B } & 0.1889(2) & 0.88031(13) & 0.84467(13) & 0.0615(5) \\ \text { H13B } & 0.110617 & 0.880890 & 0.803547 & 0.074^{*} \\ \text { C14A } & 0.0710(2) & 0.63823(14) & 0.84934(16) & 0.0687(6) \\ \text { H14A } & 0.058768 & 0.660791 & 0.897690 & 0.082^{*} \\ \text { C14B } & 0.2088(2) & 0.89785(15) & 0.93166(14) & 0.0733(6) \\ \text { H14B } & 0.143062 & 0.910500 & 0.948369 & 0.088^{*} \\ \text { C15A } & -0.01364(19) & 0.58550(15) & 0.79631(16) & 0.0728(6) \\ \text { H15A } & -0.083359 & 0.573110 & 0.808191 & 0.087^{*} \\ \text { C15B } & 0.3230(2) & 0.89684(15) & 0.99301(13) & 0.0743(6) \\ \text { H15B } & 0.335113 & 0.909440 & 1.050847 & 0.089^{*} \\ \text { C16A } & 0.00499(18) & 0.55107(15) & 0.72556(15) & 0.0713(6) \\ \text { H16A } & -0.051928 & 0.514896 & 0.689846 & 0.086^{*} \\ \text { C16B } & 0.4194(2) & 0.87709(17) & 0.96852(14) & 0.0799(7) \\ \text { H16B } & 0.497078 & 0.875101 & 1.010346 & 0.096^{*} \\ \text { C17A } & 0.10845(17) & 0.57004(13) & 0.70712(12) & 0.0580(5) \\ \text { H17A } & 0.121368 & 0.546455 & 0.659469 & 0.070^{*} \\ \text { C17B } & 0.40270(19) & 0.86009(14) & 0.88256(12) & 0.0637(5) \\ \text { H17B } & 0.468896 & 0.847365 & 0.866451 & 0.076^{*}\end{array}$

Atomic displacement parameters $\left(\AA^{2}\right)$

\begin{tabular}{lllllll}
\hline & $U^{11}$ & $U^{22}$ & $U^{33}$ & $U^{12}$ & $U^{13}$ & $U^{23}$ \\
\hline S1A & $0.0501(3)$ & $0.1132(5)$ & $0.0526(3)$ & $-0.0013(3)$ & $0.0178(2)$ & $0.0220(3)$ \\
O1A & $0.0852(10)$ & $0.0582(8)$ & $0.1095(12)$ & $0.0080(7)$ & $0.0668(10)$ & $-0.0077(8)$ \\
N1A & $0.0427(7)$ & $0.0479(8)$ & $0.0540(8)$ & $0.0015(6)$ & $0.0267(6)$ & $0.0025(6)$ \\
N1B & $0.0546(8)$ & $0.0508(8)$ & $0.0435(7)$ & $0.0033(6)$ & $0.0257(6)$ & $0.0007(6)$ \\
S1B & $0.0536(3)$ & $0.1936(8)$ & $0.0591(3)$ & $0.0382(4)$ & $0.0165(3)$ & $-0.0226(4)$ \\
O1B & $0.0907(10)$ & $0.0565(8)$ & $0.0774(9)$ & $-0.0040(7)$ & $0.0513(8)$ & $0.0100(7)$ \\
C2A & $0.0387(8)$ & $0.0476(9)$ & $0.0469(8)$ & $-0.0015(6)$ & $0.0201(7)$ & $0.0002(7)$ \\
C2B & $0.0423(9)$ & $0.0539(10)$ & $0.0380(8)$ & $0.0038(7)$ & $0.0152(7)$ & $-0.0023(7)$ \\
N3A & $0.0499(8)$ & $0.0422(7)$ & $0.0625(9)$ & $-0.0013(6)$ & $0.0304(7)$ & $-0.0013(6)$ \\
N3B & $0.0495(8)$ & $0.0443(7)$ & $0.0464(7)$ & $-0.0005(6)$ & $0.0195(6)$ & $-0.0044(6)$ \\
C4A & $0.0521(10)$ & $0.0519(10)$ & $0.0633(11)$ & $-0.0020(8)$ & $0.0333(9)$ & $-0.0068(8)$
\end{tabular}




\begin{tabular}{|c|c|c|c|c|c|c|}
\hline C4B & $0.0489(9)$ & $0.0503(9)$ & $0.0484(9)$ & $-0.0023(7)$ & $0.0229(8)$ & $0.0002(7)$ \\
\hline $\mathrm{C} 4 \mathrm{~A}^{\prime}$ & $0.0469(9)$ & $0.0512(9)$ & $0.0569(10)$ & $-0.0034(7)$ & $0.0290(8)$ & $-0.0030(7)$ \\
\hline $\mathrm{C}^{4} \mathrm{~B}^{\prime}$ & 0.0445 (9) & $0.0489(9)$ & $0.0425(8)$ & $-0.0018(7)$ & $0.0198(7)$ & $-0.0030(7)$ \\
\hline $\mathrm{C} 5 \mathrm{~A}$ & $0.0646(12)$ & $0.0689(13)$ & $0.0855(14)$ & $-0.0023(10)$ & $0.0513(11)$ & $0.0005(10)$ \\
\hline C5B & $0.0658(12)$ & 0.0649 (11) & $0.0512(10)$ & $0.0001(9)$ & $0.0335(9)$ & $-0.0045(8)$ \\
\hline C6A & 0.0704 (14) & 0.0738 (14) & $0.0899(15)$ & $-0.0128(11)$ & $0.0511(12)$ & $0.0088(11)$ \\
\hline C6B & $0.0848(15)$ & $0.0734(13)$ & $0.0648(12)$ & $0.0046(11)$ & $0.0429(11)$ & $-0.0170(10)$ \\
\hline C7A & $0.0699(13)$ & $0.0544(11)$ & $0.0843(14)$ & $-0.0078(10)$ & $0.0371(11)$ & $0.0123(10)$ \\
\hline C7B & $0.0952(17)$ & $0.0519(11)$ & $0.0867(15)$ & $0.0033(11)$ & $0.0476(13)$ & $-0.0166(10)$ \\
\hline $\mathrm{C} 8 \mathrm{~A}$ & $0.0580(11)$ & $0.0485(10)$ & $0.0733(12)$ & $0.0009(8)$ & $0.0339(10)$ & $0.0057(8)$ \\
\hline $\mathrm{C} 8 \mathrm{~B}$ & $0.0857(15)$ & $0.0480(10)$ & $0.0714(13)$ & $0.0014(9)$ & $0.0425(11)$ & $-0.0013(8)$ \\
\hline $\mathrm{C} 8 \mathrm{~A}^{\prime}$ & $0.0409(8)$ & $0.0473(9)$ & $0.0498(9)$ & $-0.0028(7)$ & $0.0222(7)$ & $0.0001(7)$ \\
\hline $\mathrm{C}^{\mathrm{B}} \mathrm{B}^{\prime}$ & $0.0504(9)$ & $0.0468(9)$ & $0.0442(8)$ & $0.0017(7)$ & $0.0227(7)$ & $-0.0018(7)$ \\
\hline C9A & $0.0849(15)$ & $0.0444(10)$ & $0.0987(16)$ & $-0.0026(10)$ & $0.0505(13)$ & $0.0025(10)$ \\
\hline C9B & $0.0868(15)$ & $0.0460(10)$ & $0.0687(12)$ & $0.0036(10)$ & $0.0270(11)$ & $-0.0114(9)$ \\
\hline $\mathrm{C} 10 \mathrm{~A}$ & $0.0412(9)$ & $0.0513(9)$ & $0.0518(9)$ & $-0.0005(7)$ & $0.0228(7)$ & $0.0051(7)$ \\
\hline $\mathrm{C} 10 \mathrm{~B}$ & $0.0501(10)$ & $0.0655(11)$ & $0.0435(9)$ & $0.0082(8)$ & $0.0203(8)$ & $-0.0063(8)$ \\
\hline N11A & $0.0415(7)$ & $0.0561(8)$ & $0.0453(8)$ & $-0.0012(6)$ & $0.0217(6)$ & $0.0100(6)$ \\
\hline N11B & $0.0475(8)$ & $0.0537(8)$ & 0.0415 (7) & $0.0071(6)$ & $0.0222(6)$ & $-0.0045(6)$ \\
\hline $\mathrm{C} 12 \mathrm{~A}$ & $0.0385(8)$ & $0.0533(9)$ & $0.0500(9)$ & $0.0032(7)$ & $0.0213(7)$ & $0.0141(7)$ \\
\hline $\mathrm{C} 12 \mathrm{~B}$ & $0.0560(10)$ & $0.0462(9)$ & $0.0415(8)$ & $0.0035(7)$ & $0.0241(8)$ & $-0.0018(6)$ \\
\hline $\mathrm{C} 13 \mathrm{~A}$ & $0.0570(11)$ & $0.0587(11)$ & $0.0684(11)$ & $0.0054(9)$ & $0.0348(9)$ & $0.0052(9)$ \\
\hline C13B & $0.0619(12)$ & $0.0770(13)$ & $0.0520(10)$ & $0.0144(10)$ & $0.0280(9)$ & $-0.0044(9)$ \\
\hline $\mathrm{C} 14 \mathrm{~A}$ & $0.0647(13)$ & $0.0808(14)$ & $0.0776(14)$ & $0.0150(11)$ & $0.0461(12)$ & $0.0154(11)$ \\
\hline C14B & $0.0842(16)$ & $0.0910(15)$ & $0.0588(12)$ & $0.0145(12)$ & $0.0426(12)$ & $-0.0071(11)$ \\
\hline C15A & $0.0501(11)$ & $0.0950(16)$ & $0.0849(15)$ & $0.0072(11)$ & $0.0380(11)$ & $0.0303(13)$ \\
\hline C15B & $0.0956(17)$ & $0.0875(15)$ & $0.0461(10)$ & $-0.0038(13)$ & $0.0329(11)$ & $-0.0109(10)$ \\
\hline C16A & $0.0486(11)$ & $0.0888(15)$ & $0.0742(13)$ & $-0.0137(10)$ & $0.0187(10)$ & $0.0193(11)$ \\
\hline $\mathrm{C} 16 \mathrm{~B}$ & $0.0736(15)$ & $0.116(2)$ & $0.0473(11)$ & $-0.0050(13)$ & $0.0174(10)$ & $-0.0126(11)$ \\
\hline C17A & $0.0507(10)$ & $0.0729(12)$ & $0.0521(10)$ & $-0.0069(9)$ & $0.0203(8)$ & $0.0098(9)$ \\
\hline C17B & $0.0556(11)$ & $0.0883(15)$ & $0.0490(10)$ & $0.0010(10)$ & $0.0207(9)$ & $-0.0083(9)$ \\
\hline
\end{tabular}

Geometric parameters $\left(\AA,{ }^{\circ}\right)$

\begin{tabular}{llll}
\hline $\mathrm{S} 1 \mathrm{~A}-\mathrm{C} 10 \mathrm{~A}$ & $1.6385(17)$ & $\mathrm{C} 8 \mathrm{~B}-\mathrm{C} 8 \mathrm{~B}^{\prime}$ & $1.399(2)$ \\
$\mathrm{O} 1 \mathrm{~A}-\mathrm{C} 4 \mathrm{~A}$ & $1.219(2)$ & $\mathrm{C} 8 \mathrm{~B}-\mathrm{H} 8 \mathrm{~B}$ & 0.9300 \\
$\mathrm{~N} 1 \mathrm{~A}-\mathrm{C} 2 \mathrm{~A}$ & $1.292(2)$ & $\mathrm{C} 9 \mathrm{~A}-\mathrm{H} 9 \mathrm{AA}$ & 0.9600 \\
$\mathrm{~N} 1 \mathrm{~A}-\mathrm{C} 8 \mathrm{~A}^{\prime}$ & $1.392(2)$ & $\mathrm{C} 9 \mathrm{~A}-\mathrm{H} 9 \mathrm{AB}$ & 0.9600 \\
$\mathrm{~N} 1 \mathrm{~B}-\mathrm{C} 2 \mathrm{~B}$ & $1.293(2)$ & $\mathrm{C} 9 \mathrm{~A}-\mathrm{H} 9 \mathrm{AC}$ & 0.9600 \\
$\mathrm{~N} 1 \mathrm{~B}-\mathrm{C} 8 \mathrm{~B}^{\prime}$ & $1.392(2)$ & $\mathrm{C} 9 \mathrm{~B}-\mathrm{H} 9 \mathrm{BA}$ & 0.9600 \\
$\mathrm{~S} 1 \mathrm{~B}-\mathrm{C} 10 \mathrm{~B}$ & $1.6401(18)$ & $\mathrm{C} 9 \mathrm{~B}-\mathrm{H} 9 \mathrm{BB}$ & 0.9600 \\
$\mathrm{O} 1 \mathrm{~B}-\mathrm{C} 4 \mathrm{~B}$ & $1.220(2)$ & $\mathrm{C} 9 \mathrm{~B}-\mathrm{H} 9 \mathrm{BC}$ & 0.9600 \\
$\mathrm{C} 2 \mathrm{~A}-\mathrm{N} 3 \mathrm{~A}$ & $1.367(2)$ & $\mathrm{C} 10 \mathrm{~A}-\mathrm{N} 11 \mathrm{~A}$ & $1.332(2)$ \\
$\mathrm{C} 2 \mathrm{~A}-\mathrm{C} 10 \mathrm{~A}$ & $1.511(2)$ & $\mathrm{C} 10 \mathrm{~B}-\mathrm{N} 11 \mathrm{~B}$ & $1.334(2)$ \\
$\mathrm{C} 2 \mathrm{~B}-\mathrm{N} 3 \mathrm{~B}$ & $1.368(2)$ & $\mathrm{N} 11 \mathrm{~A}-\mathrm{C} 12 \mathrm{~A}$ & $1.430(2)$ \\
$\mathrm{C} 2 \mathrm{~B}-\mathrm{C} 10 \mathrm{~B}$ & $1.510(2)$ & $\mathrm{N} 11 \mathrm{~A}-\mathrm{H} 11 \mathrm{~A}$ & $0.88(2)$ \\
$\mathrm{N} 3 \mathrm{~A}-\mathrm{C} 4 \mathrm{~A}$ & $1.404(2)$ & $\mathrm{N} 11 \mathrm{~B}-\mathrm{C} 12 \mathrm{~B}$ & $1.420(2)$ \\
$\mathrm{N} 3 \mathrm{~A}-\mathrm{C} 9 \mathrm{~A}$ & $1.467(2)$ & $\mathrm{N} 11 \mathrm{~B}-\mathrm{H} 11 \mathrm{~B}$ & $0.87(2)$
\end{tabular}




\begin{tabular}{|c|c|c|c|}
\hline $\mathrm{N} 3 \mathrm{~B}-\mathrm{C} 4 \mathrm{~B}$ & $1.399(2)$ & $\mathrm{C} 12 \mathrm{~A}-\mathrm{C} 17 \mathrm{~A}$ & $1.379(3)$ \\
\hline $\mathrm{N} 3 \mathrm{~B}-\mathrm{C} 9 \mathrm{~B}$ & $1.470(2)$ & $\mathrm{C} 12 \mathrm{~A}-\mathrm{C} 13 \mathrm{~A}$ & $1.382(3)$ \\
\hline $\mathrm{C} 4 \mathrm{~A}-\mathrm{C} 4 \mathrm{~A}^{\prime}$ & $1.453(3)$ & $\mathrm{C} 12 \mathrm{~B}-\mathrm{C} 13 \mathrm{~B}$ & $1.375(2)$ \\
\hline $\mathrm{C} 4 \mathrm{~B}-\mathrm{C} 4 \mathrm{~B}^{\prime}$ & $1.455(2)$ & $\mathrm{C} 12 \mathrm{~B}-\mathrm{C} 17 \mathrm{~B}$ & $1.390(3)$ \\
\hline $\mathrm{C} 4 \mathrm{~A}^{\prime}-\mathrm{C} 8 \mathrm{~A}^{\prime}$ & $1.396(2)$ & $\mathrm{C} 13 \mathrm{~A}-\mathrm{C} 14 \mathrm{~A}$ & $1.382(3)$ \\
\hline $\mathrm{C} 4 \mathrm{~A}^{\prime}-\mathrm{C} 5 \mathrm{~A}$ & $1.401(2)$ & $\mathrm{C} 13 \mathrm{~A}-\mathrm{H} 13 \mathrm{~A}$ & 0.9300 \\
\hline $\mathrm{C}^{2} \mathrm{~B}^{\prime}-\mathrm{C} 8 \mathrm{~B}^{\prime}$ & $1.392(2)$ & $\mathrm{C} 13 \mathrm{~B}-\mathrm{C} 14 \mathrm{~B}$ & $1.390(3)$ \\
\hline $\mathrm{C}_{4} \mathrm{~B}^{\prime}-\mathrm{C} 5 \mathrm{~B}$ & $1.399(2)$ & $\mathrm{C} 13 \mathrm{~B}-\mathrm{H} 13 \mathrm{~B}$ & 0.9300 \\
\hline $\mathrm{C} 5 \mathrm{~A}-\mathrm{C} 6 \mathrm{~A}$ & $1.371(3)$ & $\mathrm{C} 14 \mathrm{~A}-\mathrm{C} 15 \mathrm{~A}$ & $1.372(3)$ \\
\hline $\mathrm{C} 5 \mathrm{~A}-\mathrm{H} 5 \mathrm{~A}$ & 0.9300 & $\mathrm{C} 14 \mathrm{~A}-\mathrm{H} 14 \mathrm{~A}$ & 0.9300 \\
\hline $\mathrm{C} 5 \mathrm{~B}-\mathrm{C} 6 \mathrm{~B}$ & $1.367(3)$ & $\mathrm{C} 14 \mathrm{~B}-\mathrm{C} 15 \mathrm{~B}$ & $1.365(3)$ \\
\hline $\mathrm{C} 5 \mathrm{~B}-\mathrm{H} 5 \mathrm{~B}$ & 0.9300 & $\mathrm{C} 14 \mathrm{~B}-\mathrm{H} 14 \mathrm{~B}$ & 0.9300 \\
\hline $\mathrm{C} 6 \mathrm{~A}-\mathrm{C} 7 \mathrm{~A}$ & $1.385(3)$ & $\mathrm{C} 15 \mathrm{~A}-\mathrm{C} 16 \mathrm{~A}$ & $1.374(3)$ \\
\hline C6A-H6A & 0.9300 & $\mathrm{C} 15 \mathrm{~A}-\mathrm{H} 15 \mathrm{~A}$ & 0.9300 \\
\hline $\mathrm{C} 6 \mathrm{~B}-\mathrm{C} 7 \mathrm{~B}$ & $1.387(3)$ & $\mathrm{C} 15 \mathrm{~B}-\mathrm{C} 16 \mathrm{~B}$ & $1.369(3)$ \\
\hline $\mathrm{C} 6 \mathrm{~B}-\mathrm{H} 6 \mathrm{~B}$ & 0.9300 & $\mathrm{C} 15 \mathrm{~B}-\mathrm{H} 15 \mathrm{~B}$ & 0.9300 \\
\hline $\mathrm{C} 7 \mathrm{~A}-\mathrm{C} 8 \mathrm{~A}$ & $1.373(3)$ & $\mathrm{C} 16 \mathrm{~A}-\mathrm{C} 17 \mathrm{~A}$ & $1.389(3)$ \\
\hline $\mathrm{C} 7 \mathrm{~A}-\mathrm{H} 7 \mathrm{~A}$ & 0.9300 & $\mathrm{C} 16 \mathrm{~A}-\mathrm{H} 16 \mathrm{~A}$ & 0.9300 \\
\hline $\mathrm{C} 7 \mathrm{~B}-\mathrm{C} 8 \mathrm{~B}$ & $1.375(3)$ & $\mathrm{C} 16 \mathrm{~B}-\mathrm{C} 17 \mathrm{~B}$ & $1.380(3)$ \\
\hline $\mathrm{C} 7 \mathrm{~B}-\mathrm{H} 7 \mathrm{~B}$ & 0.9300 & $\mathrm{C} 16 \mathrm{~B}-\mathrm{H} 16 \mathrm{~B}$ & 0.9300 \\
\hline $\mathrm{C} 8 \mathrm{~A}-\mathrm{C} 8 \mathrm{~A}^{\prime}$ & $1.396(2)$ & $\mathrm{C} 17 \mathrm{~A}-\mathrm{H} 17 \mathrm{~A}$ & 0.9300 \\
\hline $\mathrm{C} 8 \mathrm{~A}-\mathrm{H} 8 \mathrm{~A}$ & 0.9300 & $\mathrm{C} 17 \mathrm{~B}-\mathrm{H} 17 \mathrm{~B}$ & 0.9300 \\
\hline $\mathrm{C} 2 \mathrm{~A}-\mathrm{N} 1 \mathrm{~A}-\mathrm{C} 8 \mathrm{~A}^{\prime}$ & $117.91(14)$ & H9AA-C9A-H9AB & 109.5 \\
\hline $\mathrm{C} 2 \mathrm{~B}-\mathrm{N} 1 \mathrm{~B}-\mathrm{C} 8 \mathrm{~B}^{\prime}$ & $117.44(14)$ & N3A-C9A-H9AC & 109.5 \\
\hline $\mathrm{N} 1 \mathrm{~A}-\mathrm{C} 2 \mathrm{~A}-\mathrm{N} 3 \mathrm{~A}$ & $124.89(14)$ & H9AA-C9A-H9AC & 109.5 \\
\hline $\mathrm{N} 1 \mathrm{~A}-\mathrm{C} 2 \mathrm{~A}-\mathrm{C} 10 \mathrm{~A}$ & $116.71(14)$ & $\mathrm{H} 9 \mathrm{AB}-\mathrm{C} 9 \mathrm{~A}-\mathrm{H} 9 \mathrm{AC}$ & 109.5 \\
\hline $\mathrm{N} 3 \mathrm{~A}-\mathrm{C} 2 \mathrm{~A}-\mathrm{C} 10 \mathrm{~A}$ & $118.39(14)$ & $\mathrm{N} 3 \mathrm{~B}-\mathrm{C} 9 \mathrm{~B}-\mathrm{H} 9 \mathrm{BA}$ & 109.5 \\
\hline $\mathrm{N} 1 \mathrm{~B}-\mathrm{C} 2 \mathrm{~B}-\mathrm{N} 3 \mathrm{~B}$ & $125.33(14)$ & $\mathrm{N} 3 \mathrm{~B}-\mathrm{C} 9 \mathrm{~B}-\mathrm{H} 9 \mathrm{BB}$ & 109.5 \\
\hline $\mathrm{N} 1 \mathrm{~B}-\mathrm{C} 2 \mathrm{~B}-\mathrm{C} 10 \mathrm{~B}$ & $116.84(15)$ & $\mathrm{H} 9 \mathrm{BA}-\mathrm{C} 9 \mathrm{~B}-\mathrm{H} 9 \mathrm{BB}$ & 109.5 \\
\hline $\mathrm{N} 3 \mathrm{~B}-\mathrm{C} 2 \mathrm{~B}-\mathrm{C} 10 \mathrm{~B}$ & $117.83(15)$ & $\mathrm{N} 3 \mathrm{~B}-\mathrm{C} 9 \mathrm{~B}-\mathrm{H} 9 \mathrm{BC}$ & 109.5 \\
\hline $\mathrm{C} 2 \mathrm{~A}-\mathrm{N} 3 \mathrm{~A}-\mathrm{C} 4 \mathrm{~A}$ & $121.39(14)$ & $\mathrm{H} 9 \mathrm{BA}-\mathrm{C} 9 \mathrm{~B}-\mathrm{H} 9 \mathrm{BC}$ & 109.5 \\
\hline $\mathrm{C} 2 \mathrm{~A}-\mathrm{N} 3 \mathrm{~A}-\mathrm{C} 9 \mathrm{~A}$ & $122.23(15)$ & $\mathrm{H} 9 \mathrm{BB}-\mathrm{C} 9 \mathrm{~B}-\mathrm{H} 9 \mathrm{BC}$ & 109.5 \\
\hline $\mathrm{C} 4 \mathrm{~A}-\mathrm{N} 3 \mathrm{~A}-\mathrm{C} 9 \mathrm{~A}$ & $116.35(15)$ & $\mathrm{N} 11 \mathrm{~A}-\mathrm{C} 10 \mathrm{~A}-\mathrm{C} 2 \mathrm{~A}$ & $112.34(14)$ \\
\hline $\mathrm{C} 2 \mathrm{~B}-\mathrm{N} 3 \mathrm{~B}-\mathrm{C} 4 \mathrm{~B}$ & $121.15(14)$ & $\mathrm{N} 11 \mathrm{~A}-\mathrm{C} 10 \mathrm{~A}-\mathrm{S} 1 \mathrm{~A}$ & $127.74(13)$ \\
\hline $\mathrm{C} 2 \mathrm{~B}-\mathrm{N} 3 \mathrm{~B}-\mathrm{C} 9 \mathrm{~B}$ & $122.32(15)$ & $\mathrm{C} 2 \mathrm{~A}-\mathrm{C} 10 \mathrm{~A}-\mathrm{S} 1 \mathrm{~A}$ & $119.91(12)$ \\
\hline $\mathrm{C} 4 \mathrm{~B}-\mathrm{N} 3 \mathrm{~B}-\mathrm{C} 9 \mathrm{~B}$ & $116.47(15)$ & $\mathrm{N} 11 \mathrm{~B}-\mathrm{C} 10 \mathrm{~B}-\mathrm{C} 2 \mathrm{~B}$ & $111.73(14)$ \\
\hline $\mathrm{O} 1 \mathrm{~A}-\mathrm{C} 4 \mathrm{~A}-\mathrm{N} 3 \mathrm{~A}$ & $120.23(17)$ & $\mathrm{N} 11 \mathrm{~B}-\mathrm{C} 10 \mathrm{~B}-\mathrm{S} 1 \mathrm{~B}$ & $130.79(13)$ \\
\hline $\mathrm{O} 1 \mathrm{~A}-\mathrm{C} 4 \mathrm{~A}-\mathrm{C} 4 \mathrm{~A}^{\prime}$ & $125.05(17)$ & $\mathrm{C} 2 \mathrm{~B}-\mathrm{C} 10 \mathrm{~B}-\mathrm{S} 1 \mathrm{~B}$ & $117.47(13)$ \\
\hline $\mathrm{N} 3 \mathrm{~A}-\mathrm{C} 4 \mathrm{~A}-\mathrm{C} 4 \mathrm{~A}^{\prime}$ & $114.72(14)$ & $\mathrm{C} 10 \mathrm{~A}-\mathrm{N} 11 \mathrm{~A}-\mathrm{C} 12 \mathrm{~A}$ & $126.80(14)$ \\
\hline $\mathrm{O} 1 \mathrm{~B}-\mathrm{C} 4 \mathrm{~B}-\mathrm{N} 3 \mathrm{~B}$ & $120.52(16)$ & $\mathrm{C} 10 \mathrm{~A}-\mathrm{N} 11 \mathrm{~A}-\mathrm{H} 11 \mathrm{~A}$ & $119.1(13)$ \\
\hline $\mathrm{O} 1 \mathrm{~B}-\mathrm{C} 4 \mathrm{~B}-\mathrm{C} 4 \mathrm{~B}^{\prime}$ & $124.74(16)$ & $\mathrm{C} 12 \mathrm{~A}-\mathrm{N} 11 \mathrm{~A}-\mathrm{H} 11 \mathrm{~A}$ & $113.5(13)$ \\
\hline $\mathrm{N} 3 \mathrm{~B}-\mathrm{C} 4 \mathrm{~B}-\mathrm{C} 4 \mathrm{~B}^{\prime}$ & $114.73(14)$ & $\mathrm{C} 10 \mathrm{~B}-\mathrm{N} 11 \mathrm{~B}-\mathrm{C} 12 \mathrm{~B}$ & $131.43(15)$ \\
\hline $\mathrm{C} 8 \mathrm{~A}^{\prime}-\mathrm{C} 4 \mathrm{~A}^{\prime}-\mathrm{C} 5 \mathrm{~A}$ & $119.71(17)$ & $\mathrm{C} 10 \mathrm{~B}-\mathrm{N} 11 \mathrm{~B}-\mathrm{H} 11 \mathrm{~B}$ & $114.1(13)$ \\
\hline $\mathrm{C} 8 \mathrm{~A}^{\prime}-\mathrm{C} 4 \mathrm{~A}^{\prime}-\mathrm{C} 4 \mathrm{~A}$ & $119.49(15)$ & $\mathrm{C} 12 \mathrm{~B}-\mathrm{N} 11 \mathrm{~B}-\mathrm{H} 11 \mathrm{~B}$ & $114.0(13)$ \\
\hline $\mathrm{C} 5 \mathrm{~A}-\mathrm{C} 4 \mathrm{~A}^{\prime}-\mathrm{C} 4 \mathrm{~A}$ & $120.80(16)$ & $\mathrm{C} 17 \mathrm{~A}-\mathrm{C} 12 \mathrm{~A}-\mathrm{C} 13 \mathrm{~A}$ & $120.63(17)$ \\
\hline $\mathrm{C} 8 \mathrm{~B}^{\prime}-\mathrm{C} 4 \mathrm{~B}^{\prime}-\mathrm{C} 5 \mathrm{~B}$ & $120.06(16)$ & $\mathrm{C} 17 \mathrm{~A}-\mathrm{C} 12 \mathrm{~A}-\mathrm{N} 11 \mathrm{~A}$ & $117.17(16)$ \\
\hline $\mathrm{C} 8 \mathrm{~B}^{\prime}-\mathrm{C} 4 \mathrm{~B}^{\prime}-\mathrm{C} 4 \mathrm{~B}$ & $119.62(14)$ & $\mathrm{C} 13 \mathrm{~A}-\mathrm{C} 12 \mathrm{~A}-\mathrm{N} 11 \mathrm{~A}$ & $122.12(17)$ \\
\hline
\end{tabular}




\begin{tabular}{|c|c|c|c|}
\hline $\mathrm{C} 5 \mathrm{~B}-\mathrm{C} 4 \mathrm{~B}^{\prime}-\mathrm{C} 4 \mathrm{~B}$ & $120.33(16)$ & $\mathrm{C} 13 \mathrm{~B}-\mathrm{C} 12 \mathrm{~B}-\mathrm{C} 17 \mathrm{~B}$ & $119.80(16)$ \\
\hline $\mathrm{C} 6 \mathrm{~A}-\mathrm{C} 5 \mathrm{~A}-\mathrm{C} 4 \mathrm{~A}^{\prime}$ & $120.07(19)$ & $\mathrm{C} 13 \mathrm{~B}-\mathrm{C} 12 \mathrm{~B}-\mathrm{N} 11 \mathrm{~B}$ & $125.03(17)$ \\
\hline $\mathrm{C} 6 \mathrm{~A}-\mathrm{C} 5 \mathrm{~A}-\mathrm{H} 5 \mathrm{~A}$ & 120.0 & $\mathrm{C} 17 \mathrm{~B}-\mathrm{C} 12 \mathrm{~B}-\mathrm{N} 11 \mathrm{~B}$ & $115.15(16)$ \\
\hline $\mathrm{C} 4 \mathrm{~A}^{\prime}-\mathrm{C} 5 \mathrm{~A}-\mathrm{H} 5 \mathrm{~A}$ & 120.0 & $\mathrm{C} 14 \mathrm{~A}-\mathrm{C} 13 \mathrm{~A}-\mathrm{C} 12 \mathrm{~A}$ & $119.0(2)$ \\
\hline $\mathrm{C} 6 \mathrm{~B}-\mathrm{C} 5 \mathrm{~B}-\mathrm{C} 4 \mathrm{~B}^{\prime}$ & $119.92(18)$ & $\mathrm{C} 14 \mathrm{~A}-\mathrm{C} 13 \mathrm{~A}-\mathrm{H} 13 \mathrm{~A}$ & 120.5 \\
\hline $\mathrm{C} 6 \mathrm{~B}-\mathrm{C} 5 \mathrm{~B}-\mathrm{H} 5 \mathrm{~B}$ & 120.0 & $\mathrm{C} 12 \mathrm{~A}-\mathrm{C} 13 \mathrm{~A}-\mathrm{H} 13 \mathrm{~A}$ & 120.5 \\
\hline $\mathrm{C} 4 \mathrm{~B}^{\prime}-\mathrm{C} 5 \mathrm{~B}-\mathrm{H} 5 \mathrm{~B}$ & 120.0 & $\mathrm{C} 12 \mathrm{~B}-\mathrm{C} 13 \mathrm{~B}-\mathrm{C} 14 \mathrm{~B}$ & $119.2(2)$ \\
\hline $\mathrm{C} 5 \mathrm{~A}-\mathrm{C} 6 \mathrm{~A}-\mathrm{C} 7 \mathrm{~A}$ & $120.17(18)$ & $\mathrm{C} 12 \mathrm{~B}-\mathrm{C} 13 \mathrm{~B}-\mathrm{H} 13 \mathrm{~B}$ & 120.4 \\
\hline $\mathrm{C} 5 \mathrm{~A}-\mathrm{C} 6 \mathrm{~A}-\mathrm{H} 6 \mathrm{~A}$ & 119.9 & $\mathrm{C} 14 \mathrm{~B}-\mathrm{C} 13 \mathrm{~B}-\mathrm{H} 13 \mathrm{~B}$ & 120.4 \\
\hline $\mathrm{C} 7 \mathrm{~A}-\mathrm{C} 6 \mathrm{~A}-\mathrm{H} 6 \mathrm{~A}$ & 119.9 & $\mathrm{C} 15 \mathrm{~A}-\mathrm{C} 14 \mathrm{~A}-\mathrm{C} 13 \mathrm{~A}$ & $121.0(2)$ \\
\hline $\mathrm{C} 5 \mathrm{~B}-\mathrm{C} 6 \mathrm{~B}-\mathrm{C} 7 \mathrm{~B}$ & $120.16(18)$ & $\mathrm{C} 15 \mathrm{~A}-\mathrm{C} 14 \mathrm{~A}-\mathrm{H} 14 \mathrm{~A}$ & 119.5 \\
\hline $\mathrm{C} 5 \mathrm{~B}-\mathrm{C} 6 \mathrm{~B}-\mathrm{H} 6 \mathrm{~B}$ & 119.9 & $\mathrm{C} 13 \mathrm{~A}-\mathrm{C} 14 \mathrm{~A}-\mathrm{H} 14 \mathrm{~A}$ & 119.5 \\
\hline $\mathrm{C} 7 \mathrm{~B}-\mathrm{C} 6 \mathrm{~B}-\mathrm{H} 6 \mathrm{~B}$ & 119.9 & $\mathrm{C} 15 \mathrm{~B}-\mathrm{C} 14 \mathrm{~B}-\mathrm{C} 13 \mathrm{~B}$ & $121.2(2)$ \\
\hline $\mathrm{C} 8 \mathrm{~A}-\mathrm{C} 7 \mathrm{~A}-\mathrm{C} 6 \mathrm{~A}$ & $120.58(19)$ & $\mathrm{C} 15 \mathrm{~B}-\mathrm{C} 14 \mathrm{~B}-\mathrm{H} 14 \mathrm{~B}$ & 119.4 \\
\hline $\mathrm{C} 8 \mathrm{~A}-\mathrm{C} 7 \mathrm{~A}-\mathrm{H} 7 \mathrm{~A}$ & 119.7 & $\mathrm{C} 13 \mathrm{~B}-\mathrm{C} 14 \mathrm{~B}-\mathrm{H} 14 \mathrm{~B}$ & 119.4 \\
\hline $\mathrm{C} 6 \mathrm{~A}-\mathrm{C} 7 \mathrm{~A}-\mathrm{H} 7 \mathrm{~A}$ & 119.7 & $\mathrm{C} 14 \mathrm{~A}-\mathrm{C} 15 \mathrm{~A}-\mathrm{C} 16 \mathrm{~A}$ & $119.72(19)$ \\
\hline $\mathrm{C} 8 \mathrm{~B}-\mathrm{C} 7 \mathrm{~B}-\mathrm{C} 6 \mathrm{~B}$ & $120.80(19)$ & $\mathrm{C} 14 \mathrm{~A}-\mathrm{C} 15 \mathrm{~A}-\mathrm{H} 15 \mathrm{~A}$ & 120.1 \\
\hline $\mathrm{C} 8 \mathrm{~B}-\mathrm{C} 7 \mathrm{~B}-\mathrm{H} 7 \mathrm{~B}$ & 119.6 & $\mathrm{C} 16 \mathrm{~A}-\mathrm{C} 15 \mathrm{~A}-\mathrm{H} 15 \mathrm{~A}$ & 120.1 \\
\hline $\mathrm{C} 6 \mathrm{~B}-\mathrm{C} 7 \mathrm{~B}-\mathrm{H} 7 \mathrm{~B}$ & 119.6 & $\mathrm{C} 14 \mathrm{~B}-\mathrm{C} 15 \mathrm{~B}-\mathrm{C} 16 \mathrm{~B}$ & $119.32(19)$ \\
\hline $\mathrm{C} 7 \mathrm{~A}-\mathrm{C} 8 \mathrm{~A}-\mathrm{C} 8 \mathrm{~A}^{\prime}$ & $120.18(18)$ & $\mathrm{C} 14 \mathrm{~B}-\mathrm{C} 15 \mathrm{~B}-\mathrm{H} 15 \mathrm{~B}$ & 120.3 \\
\hline $\mathrm{C} 7 \mathrm{~A}-\mathrm{C} 8 \mathrm{~A}-\mathrm{H} 8 \mathrm{~A}$ & 119.9 & $\mathrm{C} 16 \mathrm{~B}-\mathrm{C} 15 \mathrm{~B}-\mathrm{H} 15 \mathrm{~B}$ & 120.3 \\
\hline $\mathrm{C} 8 \mathrm{~A}^{\prime}-\mathrm{C} 8 \mathrm{~A}-\mathrm{H} 8 \mathrm{~A}$ & 119.9 & $\mathrm{C} 15 \mathrm{~A}-\mathrm{C} 16 \mathrm{~A}-\mathrm{C} 17 \mathrm{~A}$ & $120.3(2)$ \\
\hline $\mathrm{C} 7 \mathrm{~B}-\mathrm{C} 8 \mathrm{~B}-\mathrm{C} 8 \mathrm{~B}^{\prime}$ & $119.73(19)$ & $\mathrm{C} 15 \mathrm{~A}-\mathrm{C} 16 \mathrm{~A}-\mathrm{H} 16 \mathrm{~A}$ & 119.9 \\
\hline $\mathrm{C} 7 \mathrm{~B}-\mathrm{C} 8 \mathrm{~B}-\mathrm{H} 8 \mathrm{~B}$ & 120.1 & $\mathrm{C} 17 \mathrm{~A}-\mathrm{C} 16 \mathrm{~A}-\mathrm{H} 16 \mathrm{~A}$ & 119.9 \\
\hline $\mathrm{C} 8 \mathrm{~B}^{\prime}-\mathrm{C} 8 \mathrm{~B}-\mathrm{H} 8 \mathrm{~B}$ & 120.1 & $\mathrm{C} 15 \mathrm{~B}-\mathrm{C} 16 \mathrm{~B}-\mathrm{C} 17 \mathrm{~B}$ & $120.8(2)$ \\
\hline $\mathrm{N} 1 \mathrm{~A}-\mathrm{C} 8 \mathrm{~A}^{\prime}-\mathrm{C}^{\prime} \mathrm{A}^{\prime}$ & $121.56(15)$ & $\mathrm{C} 15 \mathrm{~B}-\mathrm{C} 16 \mathrm{~B}-\mathrm{H} 16 \mathrm{~B}$ & 119.6 \\
\hline $\mathrm{N} 1 \mathrm{~A}-\mathrm{C} 8 \mathrm{~A}^{\prime}-\mathrm{C} 8 \mathrm{~A}$ & $119.17(15)$ & $\mathrm{C} 17 \mathrm{~B}-\mathrm{C} 16 \mathrm{~B}-\mathrm{H} 16 \mathrm{~B}$ & 119.6 \\
\hline $\mathrm{C}_{4} \mathrm{~A}^{\prime}-\mathrm{C} 8 \mathrm{~A}^{\prime}-\mathrm{C} 8 \mathrm{~A}$ & $119.26(16)$ & $\mathrm{C} 12 \mathrm{~A}-\mathrm{C} 17 \mathrm{~A}-\mathrm{C} 16 \mathrm{~A}$ & $119.35(19)$ \\
\hline $\mathrm{C}^{\prime} \mathrm{B}^{\prime}-\mathrm{C} 8 \mathrm{~B}^{\prime}-\mathrm{N} 1 \mathrm{~B}$ & $121.68(15)$ & $\mathrm{C} 12 \mathrm{~A}-\mathrm{C} 17 \mathrm{~A}-\mathrm{H} 17 \mathrm{~A}$ & 120.3 \\
\hline $\mathrm{C}^{\prime} \mathrm{B}^{\prime}-\mathrm{C} 8 \mathrm{~B}^{\prime}-\mathrm{C} 8 \mathrm{~B}$ & $119.33(16)$ & $\mathrm{C} 16 \mathrm{~A}-\mathrm{C} 17 \mathrm{~A}-\mathrm{H} 17 \mathrm{~A}$ & 120.3 \\
\hline $\mathrm{N} 1 \mathrm{~B}-\mathrm{C} 8 \mathrm{~B}^{\prime}-\mathrm{C} 8 \mathrm{~B}$ & $118.99(16)$ & $\mathrm{C} 16 \mathrm{~B}-\mathrm{C} 17 \mathrm{~B}-\mathrm{C} 12 \mathrm{~B}$ & $119.6(2)$ \\
\hline $\mathrm{N} 3 \mathrm{~A}-\mathrm{C} 9 \mathrm{~A}-\mathrm{H} 9 \mathrm{AA}$ & 109.5 & $\mathrm{C} 16 \mathrm{~B}-\mathrm{C} 17 \mathrm{~B}-\mathrm{H} 17 \mathrm{~B}$ & 120.2 \\
\hline $\mathrm{N} 3 \mathrm{~A}-\mathrm{C} 9 \mathrm{~A}-\mathrm{H} 9 \mathrm{AB}$ & 109.5 & $\mathrm{C} 12 \mathrm{~B}-\mathrm{C} 17 \mathrm{~B}-\mathrm{H} 17 \mathrm{~B}$ & 120.2 \\
\hline $\mathrm{C} 8 \mathrm{~A}^{\prime}-\mathrm{N} 1 \mathrm{~A}-\mathrm{C} 2 \mathrm{~A}-\mathrm{N} 3 \mathrm{~A}$ & $-1.0(3)$ & $\mathrm{C} 4 \mathrm{~A}-\mathrm{C} 4 \mathrm{~A}^{\prime}-\mathrm{C} 8 \mathrm{~A}^{\prime}-\mathrm{C} 8 \mathrm{~A}$ & $-178.35(17)$ \\
\hline $\mathrm{C} 8 \mathrm{~A}^{\prime}-\mathrm{N} 1 \mathrm{~A}-\mathrm{C} 2 \mathrm{~A}-\mathrm{C} 10 \mathrm{~A}$ & $179.14(14)$ & $\mathrm{C} 7 \mathrm{~A}-\mathrm{C} 8 \mathrm{~A}-\mathrm{C} 8 \mathrm{~A}^{\prime}-\mathrm{N} 1 \mathrm{~A}$ & $179.63(18)$ \\
\hline $\mathrm{C} 8 \mathrm{~B}^{\prime}-\mathrm{N} 1 \mathrm{~B}-\mathrm{C} 2 \mathrm{~B}-\mathrm{N} 3 \mathrm{~B}$ & $-1.0(3)$ & $\mathrm{C} 7 \mathrm{~A}-\mathrm{C} 8 \mathrm{~A}-\mathrm{C} 8 \mathrm{~A}^{\prime}-\mathrm{C} 4 \mathrm{~A}^{\prime}$ & $-0.4(3)$ \\
\hline $\mathrm{C} 8 \mathrm{~B}^{\prime}-\mathrm{N} 1 \mathrm{~B}-\mathrm{C} 2 \mathrm{~B}-\mathrm{C} 10 \mathrm{~B}$ & $179.33(14)$ & $\mathrm{C} 5 \mathrm{~B}-\mathrm{C} 4 \mathrm{~B}^{\prime}-\mathrm{C} 8 \mathrm{~B}^{\prime}-\mathrm{N} 1 \mathrm{~B}$ & $-179.59(16)$ \\
\hline $\mathrm{N} 1 \mathrm{~A}-\mathrm{C} 2 \mathrm{~A}-\mathrm{N} 3 \mathrm{~A}-\mathrm{C} 4 \mathrm{~A}$ & $0.6(3)$ & $\mathrm{C} 4 \mathrm{~B}-\mathrm{C} 4 \mathrm{~B}^{\prime}-\mathrm{C} 8 \mathrm{~B}^{\prime}-\mathrm{N} 1 \mathrm{~B}$ & $0.4(3)$ \\
\hline $\mathrm{C} 10 \mathrm{~A}-\mathrm{C} 2 \mathrm{~A}-\mathrm{N} 3 \mathrm{~A}-\mathrm{C} 4 \mathrm{~A}$ & $-179.57(16)$ & $\mathrm{C} 5 \mathrm{~B}-\mathrm{C}^{\prime} \mathrm{B}^{\prime}-\mathrm{C} 8 \mathrm{~B}^{\prime}-\mathrm{C} 8 \mathrm{~B}$ & $0.6(3)$ \\
\hline $\mathrm{N} 1 \mathrm{~A}-\mathrm{C} 2 \mathrm{~A}-\mathrm{N} 3 \mathrm{~A}-\mathrm{C} 9 \mathrm{~A}$ & $-177.51(18)$ & $\mathrm{C} 4 \mathrm{~B}-\mathrm{C} 4 \mathrm{~B}^{\prime}-\mathrm{C} 8 \mathrm{~B}^{\prime}-\mathrm{C} 8 \mathrm{~B}$ & $-179.50(18)$ \\
\hline $\mathrm{C} 10 \mathrm{~A}-\mathrm{C} 2 \mathrm{~A}-\mathrm{N} 3 \mathrm{~A}-\mathrm{C} 9 \mathrm{~A}$ & $2.4(3)$ & $\mathrm{C} 2 \mathrm{~B}-\mathrm{N} 1 \mathrm{~B}-\mathrm{C} 8 \mathrm{~B}^{\prime}-\mathrm{C} 4 \mathrm{~B}^{\prime}$ & $-0.5(2)$ \\
\hline $\mathrm{N} 1 \mathrm{~B}-\mathrm{C} 2 \mathrm{~B}-\mathrm{N} 3 \mathrm{~B}-\mathrm{C} 4 \mathrm{~B}$ & $2.7(3)$ & $\mathrm{C} 2 \mathrm{~B}-\mathrm{N} 1 \mathrm{~B}-\mathrm{C} 8 \mathrm{~B}^{\prime}-\mathrm{C} 8 \mathrm{~B}$ & $179.35(18)$ \\
\hline $\mathrm{C} 10 \mathrm{~B}-\mathrm{C} 2 \mathrm{~B}-\mathrm{N} 3 \mathrm{~B}-\mathrm{C} 4 \mathrm{~B}$ & $-177.67(15)$ & $\mathrm{C} 7 \mathrm{~B}-\mathrm{C} 8 \mathrm{~B}-\mathrm{C} 8 \mathrm{~B}^{\prime}-\mathrm{C} 4 \mathrm{~B}^{\prime}$ & $-0.6(3)$ \\
\hline $\mathrm{N} 1 \mathrm{~B}-\mathrm{C} 2 \mathrm{~B}-\mathrm{N} 3 \mathrm{~B}-\mathrm{C} 9 \mathrm{~B}$ & $179.73(17)$ & $\mathrm{C} 7 \mathrm{~B}-\mathrm{C} 8 \mathrm{~B}-\mathrm{C} 8 \mathrm{~B}^{\prime}-\mathrm{N} 1 \mathrm{~B}$ & $179.5(2)$ \\
\hline $\mathrm{C} 10 \mathrm{~B}-\mathrm{C} 2 \mathrm{~B}-\mathrm{N} 3 \mathrm{~B}-\mathrm{C} 9 \mathrm{~B}$ & $-0.6(2)$ & $\mathrm{N} 1 \mathrm{~A}-\mathrm{C} 2 \mathrm{~A}-\mathrm{C} 10 \mathrm{~A}-\mathrm{N} 11 \mathrm{~A}$ & $75.1(2)$ \\
\hline $\mathrm{C} 2 \mathrm{~A}-\mathrm{N} 3 \mathrm{~A}-\mathrm{C} 4 \mathrm{~A}-\mathrm{O} 1 \mathrm{~A}$ & $-178.66(18)$ & $\mathrm{N} 3 \mathrm{~A}-\mathrm{C} 2 \mathrm{~A}-\mathrm{C} 10 \mathrm{~A}-\mathrm{N} 11 \mathrm{~A}$ & $-104.80(18)$ \\
\hline $\mathrm{C} 9 \mathrm{~A}-\mathrm{N} 3 \mathrm{~A}-\mathrm{C} 4 \mathrm{~A}-\mathrm{O} 1 \mathrm{~A}$ & $-0.5(3)$ & $\mathrm{N} 1 \mathrm{~A}-\mathrm{C} 2 \mathrm{~A}-\mathrm{C} 10 \mathrm{~A}-\mathrm{S} 1 \mathrm{~A}$ & $-103.97(17)$ \\
\hline
\end{tabular}




$\begin{array}{ll}\mathrm{C} 2 \mathrm{~A}-\mathrm{N} 3 \mathrm{~A}-\mathrm{C} 4 \mathrm{~A}-\mathrm{C} 4 \mathrm{~A}^{\prime} & 0.9(3) \\ \mathrm{C} 9 \mathrm{~A}-\mathrm{N} 3 \mathrm{~A}-\mathrm{C} 4 \mathrm{~A}-\mathrm{C} 4 \mathrm{~A}^{\prime} & 179.10(17) \\ \mathrm{C} 2 \mathrm{~B}-\mathrm{N} 3 \mathrm{~B}-\mathrm{C} 4 \mathrm{~B}-\mathrm{O} 1 \mathrm{~B} & 178.41(17) \\ \mathrm{C} 9 \mathrm{~B}-\mathrm{N} 3 \mathrm{~B}-\mathrm{C} 4 \mathrm{~B}-\mathrm{O} 1 \mathrm{~B} & 1.2(3) \\ \mathrm{C} 2 \mathrm{~B}-\mathrm{N} 3 \mathrm{~B}-\mathrm{C} 4 \mathrm{~B}-\mathrm{C} 4 \mathrm{~B}^{\prime} & -2.6(2) \\ \mathrm{C} 9 \mathrm{~B}-\mathrm{N} 3 \mathrm{~B}-\mathrm{C} 4 \mathrm{~B}-\mathrm{C} 4 \mathrm{~B}^{\prime} & -179.78(16) \\ \mathrm{O} 1 \mathrm{~A}-\mathrm{C} 4 \mathrm{~A}-\mathrm{C} 4 \mathrm{~A}^{\prime}-\mathrm{C} 8 \mathrm{~A}^{\prime} & 177.6(2) \\ \mathrm{N} 3 \mathrm{~A}-\mathrm{C} 4 \mathrm{~A}-\mathrm{C} 4 \mathrm{~A}^{\prime}-\mathrm{C} 8 \mathrm{~A}^{\prime} & -1.9(3) \\ \mathrm{O} 1 \mathrm{~A}-\mathrm{C} 4 \mathrm{~A}-\mathrm{C} 4 \mathrm{~A}^{\prime}-\mathrm{C} 5 \mathrm{~A} & -2.2(3) \\ \mathrm{N} 3 \mathrm{~A}-\mathrm{C} 4 \mathrm{~A}-\mathrm{C} 4 \mathrm{~A}^{\prime}-\mathrm{C} 5 \mathrm{~A} & 178.19(18) \\ \mathrm{O} 1 \mathrm{~B}-\mathrm{C} 4 \mathrm{~B}-\mathrm{C} 4 \mathrm{~B}^{\prime}-\mathrm{C} 8 \mathrm{~B}^{\prime} & -179.87(18) \\ \mathrm{N} 3 \mathrm{~B}-\mathrm{C} 4 \mathrm{~B}-\mathrm{C} 4 \mathrm{~B}^{\prime}-\mathrm{C} 8 \mathrm{~B}^{\prime} & 1.1(2) \\ \mathrm{O} 1 \mathrm{~B}-\mathrm{C} 4 \mathrm{~B}-\mathrm{C} 4 \mathrm{~B}^{\prime}-\mathrm{C} 5 \mathrm{~B} & 0.1(3) \\ \mathrm{N} 3 \mathrm{~B}-\mathrm{C} 4 \mathrm{~B}-\mathrm{C} 4 \mathrm{~B}^{\prime}-\mathrm{C} 5 \mathrm{~B} & -178.91(16) \\ \mathrm{C} 8 \mathrm{~A}^{\prime}-\mathrm{C} 4 \mathrm{~A}^{\prime}-\mathrm{C} 5 \mathrm{~A}-\mathrm{C} 6 \mathrm{~A} & -1.4(3) \\ \mathrm{C} 4 \mathrm{~A}-\mathrm{C} 4 \mathrm{~A}^{\prime}-\mathrm{C} 5 \mathrm{~A}-\mathrm{C} 6 \mathrm{~A} & 178.5(2) \\ \mathrm{C} 8 \mathrm{~B}^{\prime}-\mathrm{C} 4 \mathrm{~B}^{\prime}-\mathrm{C} 5 \mathrm{~B}-\mathrm{C} 6 \mathrm{~B} & -0.4(3) \\ \mathrm{C} 4 \mathrm{~B}-\mathrm{C} 4 \mathrm{~B}^{\prime}-\mathrm{C} 5 \mathrm{~B}-\mathrm{C} 6 \mathrm{~B} & 179.66(18) \\ \mathrm{C} 4 \mathrm{~A}^{\prime}-\mathrm{C} 5 \mathrm{~A}-\mathrm{C} 6 \mathrm{~A}-\mathrm{C} 7 \mathrm{~A} & 0.1(4) \\ \mathrm{C} 4 \mathrm{~B}^{\prime}-\mathrm{C} 5 \mathrm{~B}-\mathrm{C} 6 \mathrm{~B}-\mathrm{C} 7 \mathrm{~B} & 0.3(3) \\ \mathrm{C} 5 \mathrm{~A}-\mathrm{C} 6 \mathrm{~A}-\mathrm{C} 7 \mathrm{~A}-\mathrm{C} 8 \mathrm{~A} & 1.1(4) \\ \mathrm{C} 5 \mathrm{~B}-\mathrm{C} 6 \mathrm{~B}-\mathrm{C} 7 \mathrm{~B}-\mathrm{C} 8 \mathrm{~B} & -0.3(4) \\ \mathrm{C} 6 \mathrm{~A}-\mathrm{C} 7 \mathrm{~A}-\mathrm{C} 8 \mathrm{~A}-\mathrm{C} 8 \mathrm{~A}^{\prime} & -0.9(3) \\ \mathrm{C} 6 \mathrm{~B}-\mathrm{C} 7 \mathrm{~B}-\mathrm{C} 8 \mathrm{~B}-\mathrm{C} 8 \mathrm{~B}^{\prime} & 0.5(4) \\ \mathrm{C} 2 \mathrm{~A}-\mathrm{N} 1 \mathrm{~A}-\mathrm{C} 8 \mathrm{~A}^{\prime}-\mathrm{C} 4 \mathrm{~A}^{\prime} & -0.1(2) \\ \mathrm{C} 2 \mathrm{~A}-\mathrm{N} 1 \mathrm{~A}-\mathrm{C} 8 \mathrm{~A}^{\prime}-\mathrm{C} 8 \mathrm{~A} & 179.82(16) \\ \mathrm{C} 5 \mathrm{~A}-\mathrm{C} 4 \mathrm{~A}^{\prime}-\mathrm{C} 8 \mathrm{~A}^{\prime}-\mathrm{N} 1 \mathrm{~A} & -178.49(18) \\ \mathrm{C} 4 \mathrm{~A}-\mathrm{C} 4 \mathrm{~A}^{\prime}-\mathrm{C} 8 \mathrm{~A}^{\prime}-\mathrm{N} 1 \mathrm{~A} & 1.6(3) \\ \mathrm{C} 5 \mathrm{~A}-\mathrm{C} 4 \mathrm{~A}^{\prime}-\mathrm{C} 8 \mathrm{~A}^{\prime}-\mathrm{C} 8 \mathrm{~A} & 1.5(3) \\ & \end{array}$

$\begin{array}{ll}\mathrm{N} 3 \mathrm{~A}-\mathrm{C} 2 \mathrm{~A}-\mathrm{C} 10 \mathrm{~A}-\mathrm{S} 1 \mathrm{~A} & 76.14(19) \\ \mathrm{N} 1 \mathrm{~B}-\mathrm{C} 2 \mathrm{~B}-\mathrm{C} 10 \mathrm{~B}-\mathrm{N} 11 \mathrm{~B} & 83.6(2) \\ \mathrm{N} 3 \mathrm{~B}-\mathrm{C} 2 \mathrm{~B}-\mathrm{C} 10 \mathrm{~B}-\mathrm{N} 11 \mathrm{~B} & -96.12(19) \\ \mathrm{N} 1 \mathrm{~B}-\mathrm{C} 2 \mathrm{~B}-\mathrm{C} 10 \mathrm{~B}-\mathrm{S} 1 \mathrm{~B} & -96.53(18) \\ \mathrm{N} 3 \mathrm{~B}-\mathrm{C} 2 \mathrm{~B}-\mathrm{C} 10 \mathrm{~B}-\mathrm{S} 1 \mathrm{~B} & 83.78(19) \\ \mathrm{C} 2 \mathrm{~A}-\mathrm{C} 10 \mathrm{~A}-\mathrm{N} 11 \mathrm{~A}-\mathrm{C} 12 \mathrm{~A} & -177.47(15) \\ \mathrm{S} 1 \mathrm{~A}-\mathrm{C} 10 \mathrm{~A}-\mathrm{N} 11 \mathrm{~A}-\mathrm{C} 12 \mathrm{~A} & 1.5(3) \\ \mathrm{C} 2 \mathrm{~B}-\mathrm{C} 10 \mathrm{~B}-\mathrm{N} 11 \mathrm{~B}-\mathrm{C} 12 \mathrm{~B} & -179.09(17) \\ \mathrm{S} 1 \mathrm{~B}-\mathrm{C} 10 \mathrm{~B}-\mathrm{N} 11 \mathrm{~B}-\mathrm{C} 12 \mathrm{~B} & 1.0(3) \\ \mathrm{C} 10 \mathrm{~A}-\mathrm{N} 11 \mathrm{~A}-\mathrm{C} 12 \mathrm{~A}-\mathrm{C} 17 \mathrm{~A} & -133.70(19) \\ \mathrm{C} 10 \mathrm{~A}-\mathrm{N} 11 \mathrm{~A}-\mathrm{C} 12 \mathrm{~A}-\mathrm{C} 13 \mathrm{~A} & 49.3(3) \\ \mathrm{C} 10 \mathrm{~B}-\mathrm{N} 11 \mathrm{~B}-\mathrm{C} 12 \mathrm{~B}-\mathrm{C} 13 \mathrm{~B} & 5.4(3) \\ \mathrm{C} 10 \mathrm{~B}-\mathrm{N} 11 \mathrm{~B}-\mathrm{C} 12 \mathrm{~B}-\mathrm{C} 17 \mathrm{~B} & -176.2(2) \\ \mathrm{C} 17 \mathrm{~A}-\mathrm{C} 12 \mathrm{~A}-\mathrm{C} 13 \mathrm{~A}-\mathrm{C} 14 \mathrm{~A} & -0.8(3) \\ \mathrm{N} 11 \mathrm{~A}-\mathrm{C} 12 \mathrm{~A}-\mathrm{C} 13 \mathrm{~A}-\mathrm{C} 14 \mathrm{~A} & 176.03(17) \\ \mathrm{C} 17 \mathrm{~B}-\mathrm{C} 12 \mathrm{~B}-\mathrm{C} 13 \mathrm{~B}-\mathrm{C} 14 \mathrm{~B} & 1.1(3) \\ \text { N11B-C12B-C13B-C14B } & 179.36(19) \\ \mathrm{C} 12 \mathrm{~A}-\mathrm{C} 13 \mathrm{~A}-\mathrm{C} 14 \mathrm{~A}-\mathrm{C} 15 \mathrm{~A} & -0.3(3) \\ \mathrm{C} 12 \mathrm{~B}-\mathrm{C} 13 \mathrm{~B}-\mathrm{C} 14 \mathrm{~B}-\mathrm{C} 15 \mathrm{~B} & -0.4(4) \\ \mathrm{C} 13 \mathrm{~A}-\mathrm{C} 14 \mathrm{~A}-\mathrm{C} 15 \mathrm{~A}-\mathrm{C} 16 \mathrm{~A} & 1.0(3) \\ \mathrm{C} 13 \mathrm{~B}-\mathrm{C} 14 \mathrm{~B}-\mathrm{C} 15 \mathrm{~B}-\mathrm{C} 16 \mathrm{~B} & -0.9(4) \\ \mathrm{C} 14 \mathrm{~A}-\mathrm{C} 15 \mathrm{~A}-\mathrm{C} 16 \mathrm{~A}-\mathrm{C} 17 \mathrm{~A} & -0.6(3) \\ \mathrm{C} 14 \mathrm{~B}-\mathrm{C} 15 \mathrm{~B}-\mathrm{C} 16 \mathrm{~B}-\mathrm{C} 17 \mathrm{~B} & 1.5(4) \\ \mathrm{C} 13 \mathrm{~A}-\mathrm{C} 12 \mathrm{~A}-\mathrm{C} 17 \mathrm{~A}-\mathrm{C} 16 \mathrm{~A} & 1.2(3) \\ \text { N11A-C12A-C17A-C16A } & -175.84(17) \\ \mathrm{C} 15 \mathrm{~A}-\mathrm{C} 16 \mathrm{~A}-\mathrm{C} 17 \mathrm{~A}-\mathrm{C} 12 \mathrm{~A} & -0.4(3) \\ \mathrm{C} 15 \mathrm{~B}-\mathrm{C} 16 \mathrm{~B}-\mathrm{C} 17 \mathrm{~B}-\mathrm{C} 12 \mathrm{~B} & -0.8(4) \\ \mathrm{C} 13 \mathrm{~B}-\mathrm{C} 12 \mathrm{~B}-\mathrm{C} 17 \mathrm{~B}-\mathrm{C} 16 \mathrm{~B} & -0.5(3) \\ \text { N11B-C12B-C17B-C16B } & -179.0(2) \\ & \end{array}$

Hydrogen-bond geometry $\left(\AA,{ }^{o}\right)$

\begin{tabular}{lllll}
\hline$D-\mathrm{H} \cdots A$ & $D-\mathrm{H}$ & $\mathrm{H} \cdots A$ & $D \cdots A$ & $D-\mathrm{H} \cdots A$ \\
\hline $\mathrm{N} 11 A-\mathrm{H} 11 A \cdots \mathrm{N} 1 B$ & $0.88(2)$ & $2.05(2)$ & $2.913(2)$ & $166.7(18)$ \\
$\mathrm{N} 11 B-\mathrm{H} 11 B \cdots \mathrm{N} 1 A$ & $0.87(2)$ & $2.04(2)$ & $2.907(2)$ & $171.6(19)$ \\
$\mathrm{C} 9 A-\mathrm{H} 9 A B \cdots \mathrm{S} 1 A$ & 0.96 & 2.87 & $3.424(2)$ & 118 \\
$\mathrm{C} 13 B-\mathrm{H} 13 B \cdots \mathrm{S} 1 B$ & 0.93 & 2.58 & $3.243(3)$ & 129 \\
$\mathrm{C} 7 A-\mathrm{H} 7 A^{\cdots} \cdots \mathrm{O} 1 B^{\mathrm{i}}$ & 0.93 & 2.49 & $3.386(3)$ & 162 \\
$\mathrm{C} 7 B-\mathrm{H} 7 B \cdots \mathrm{O} 1 A^{\mathrm{ii}}$ & 0.93 & 2.47 & $3.385(3)$ & 166 \\
\hline
\end{tabular}

Symmetry codes: (i) $-x+1,-y+2,-z+1$; (ii) $-x+1,-y+1,-z+1$. 